ARTICLE

\title{
Exon junction complex dependent mRNA localization is linked to centrosome organization during ciliogenesis
}

\author{
Oh Sung Kwon (10) 1, Rahul Mishra (10 1,4, Adham Safieddine (10 2,3, Emeline Coleno ${ }^{2,3}$, Quentin Alasseur ${ }^{1}$, \\ Marion Faucourt ${ }^{1}$, Isabelle Barbosa ${ }^{1}$, Edouard Bertrand (10) ${ }^{2,3}$, Nathalie Spassky ${ }^{1}$ \& Hervé Le Hir ${ }^{1 凶}$
}

Exon junction complexes (EJCS) mark untranslated spliced mRNAs and are crucial for the mRNA lifecycle. An imbalance in EJC dosage alters mouse neural stem cell (mNSC) division and is linked to human neurodevelopmental disorders. In quiescent mNSC and immortalized human retinal pigment epithelial (RPE1) cells, centrioles form a basal body for ciliogenesis. Here, we report that EJCs accumulate at basal bodies of mNSC or RPE1 cells and decline when these cells differentiate or resume growth. A high-throughput smFISH screen identifies two transcripts accumulating at centrosomes in quiescent cells, NIN and BICD2. In contrast to $B I C D 2$, the localization of NIN transcripts is EJC-dependent. NIN mRNA encodes a core component of centrosomes required for microtubule nucleation and anchoring. We find that EJC down-regulation impairs both pericentriolar material organization and ciliogenesis. An EJC-dependent mRNA trafficking towards centrosome and basal bodies might contribute to proper mNSC division and brain development.

\footnotetext{
${ }^{1}$ Institut de Biologie de I'Ecole Normale Supérieure (IBENS), Ecole Normale Supérieure, CNRS, INSERM, PSL Research University, Paris, France. ${ }^{2}$ Institut de Génétique Moléculaire de Montpellier, University of Montpellier, CNRS, Montpellier, France. ${ }^{3}$ Equipe labélisée Ligue Nationale Contre le Cancer, University of Montpellier, CNRS, Montpellier, France. ${ }^{4}$ Present address: Institute of Parasitology, Biology Centre, Czech Academy of Sciences, Ceske Budejovice, Czech Republic. ${ }^{凶}$ email: lehir@ens.fr
} 
$\mathrm{M}$ essenger RNAs result from a succession of maturation steps that modify transcript extremities and excise introns. These processes are tightly coupled to the transcription machinery, and ultimately lead to the packaging of mature mRNAs into large ribonucleoparticles composed of numerous RNA-binding proteins (RBPs) ${ }^{1}$. Each messenger ribonucleoprotein (mRNP) particle is composed of ubiquitous RBPs including cap-binding proteins, exon junction complexes (EJC), and polyA-binding proteins, as well as hundreds of additional common and cell-specific $\mathrm{RBPs}^{2-6}$. These RBPs densely pack the mRNP particles ${ }^{7,8}$ and govern the fate and the functions of mRNAs ${ }^{1}$.

EJCs are deposited upstream exon-exon junctions by the splicing machinery and are potentially present in multiple copies along transcripts ${ }^{9,10}$. The EJC core complex is composed of four proteins: the RNA helicase eIF4A3 (eukaryotic initiation factor $4 \mathrm{~A} 3$ or DDX48), the heterodimer MAGOH/Y14 (or RBM8) and MLN51 (Metastatic Lymph Node 51 or CASC3) ${ }^{11,12}$. At the center, eIF4A3 clamps RNA to ensure an unusually stable binding 13,14 . A dozen of additional factors bind directly or indirectly the EJC core and constitute EJC peripheral factors ${ }^{15}$. mRNP particles are largely remodeled upon translation in the cytoplasm ${ }^{7,8}$ and EJCs are disassembled at this step by scanning ribosomes ${ }^{16}$. Therefore, EJCs mark a precise period in the mRNA lifecycle between nuclear splicing and cytosolic translation. During this period, EJCs contribute to splicing regulation and to the recruitment of nuclear export factors ${ }^{15,17}$. In the cytoplasm, EJCs are intimately linked to mRNA translation and stability. First, EJCs enhance the translational efficiency of newly made mRNP by communicating with the translation machinery $15,18,19$. Second, EJCs serve as a signal for nonsense-mediated mRNA decay (NMD), when translation termination occurs before the last exon-exon junction. Thus, NMD couples the translation and degradation machineries to eliminate transcripts encoding truncated proteins or to regulate the stability of specific transcript in a translation-dependent manner ${ }^{20}$.

The implication of EJCs in several crucial steps of gene expression explains why its components are essential for cellular viability $^{21}$. In several organisms, a precise dosage of EJC components is required for proper development ${ }^{22-28}$. In humans, mutations leading to hypomorphic expression of Y14 and eIF4A3 are associated to two distinct syndromes with common neurodevelopmental phenotypes ${ }^{29}$. The thrombocytopenia with absent radius (TAR) syndrome is associated with a reduction of Y14 expression and it presents some defects in limb development and platelet production ${ }^{30}$. In the case of eIF4A3, it is linked to the autosomal recessive Richieri-Costa-Pereira syndrome (RCPS) presenting both limb and craniofacial dysmorphisms ${ }^{31}$. Copy number variants of EJC and NMD factors were also found in patients with intellectual disabilities ${ }^{32}$. A major step in understanding the link between EJC dosage effect and brain development and function in mammals derived from mouse genetics. A pioneer mutagenesis screen unraveled that MAGOH haploinsufficiency results in smaller body size and microcephaly by regulating division of neural stem cells (NSC) ${ }^{33}$. A conditional Magoh allelic knock-out leading to NSC-specific reduction in MAGOH expression confirmed its importance for cortical development. In these cells, NSC mitosis is delayed, leading to a decrease of intermediary progenitors (IP), a premature generation of neurons and an increased apoptosis of their progeny ${ }^{33-35}$. Remarkably, the generation of $R b m 8 a$ (encoding Y14) as well as eIF4A3 conditional haplo-insufficiency in mNSC phenocopied the effects observed with Magoh on embryonic neurogenesis, with a notable microcephaly ${ }^{36,37}$. However, a $M \ln 51$ conditional haploinsufficiency only partially phenocopied the three other EJC core components with less profound neurodevelopmental disorders, suggesting a more tissue-specific involvement of MLN51 $1^{38}$. EJC-associated NMD factors have also been associated to NSC maintenance and differentiation ${ }^{39-41}$. A proper dosage of fully assembled EJCs, and not only its free components, is thus clearly essential for NSC division, differentiation and brain development. However, the precise mechanisms at play remain elusive.

These observations prompted us to study EJC core proteins in primary cultures of radial glial $\mathrm{mNSC}$, which are quiescent monociliated cells. Centrosomes are composed of a pair of centrioles and a matrix of pericentriolar material (PCM) that nucleates microtubules and participates in cell cycle and signaling regulation ${ }^{42}$. When cells exit the cell cycle, the centriole pair migrates to the cell surface, and the mother centriole constitutes a basal body for primary cilium formation ${ }^{42}$.

In this work, we observe that EJC core proteins concentrate around centrosomes at the base of primary cilia both in mNSCs and human retinal pigment epithelial (RPE1) cells. This centrosomal accumulation of EJC proteins is predominant during the quiescent state as it diminishes upon cell differentiation or cellcycle re-entry. The accumulation of EJC complexes around centrosomes is RNA-dependent and ensured by a microtubuledependent pathway. A single molecule FISH (smFISH) screen identifies two mRNAs, NIN and BICD2 localizing at centrosomes in quiescent RPE1 cells. Remarkably, both EJC and translation are essential for NIN mRNA localization. Down-regulation of EJC impaired ciliogenesis and organization of the PCM, establishing a potential link between the molecular and physiological functions of the EJC.

\section{Results}

EIF4A3 and Y14 label centrosomes in quiescent mNSC. Reduced expression of any of the EJC core components in mice induces defects in NSC division and differentiation ${ }^{29}$. This prompted us to study the expression of EJC core proteins in mNSCs. We first investigated primary cultures of glial progenitors isolated from newborn mouse forebrain ${ }^{43}$. Upon serum starvation, quiescent mono-ciliated radial glial cells differentiate into ependymal cells ${ }^{44}$. Ependymal cells are multi-ciliated and are present at the surface of brain ventricles. Beating of their cilia contributes to the flow of cerebrospinal fluid. In radial glial cells, the primary cilium grows from the basal body docked at the membrane. During differentiation, amplification of centrioles leads to the production of multiple cilia at the surface of ependymal cells ${ }^{45}$.

Antibodies against FGFR1 Oncogene Partner (FOP) label the distal end of centrioles of mono and multiciliated cells and the pericentriolar area ${ }^{46,47}$, whereas antibodies against polyglutamylated tubulin decorate both centrioles and cilia ${ }^{48}$. Both antibodies clearly distinguished the mono- (Fig. 1a, c) and multi-ciliated (Fig. 1b, d) states of mNSCs and ependymal cells, respectively. We investigated the localization of the EJC core components eIF4A3 and Y14. As previously observed in other cells ${ }^{49-51}$, eIF4A3 and Y14 were mainly nuclear in both mono-ciliated and multi-ciliated mNSCs (Fig. 1a-d). However, we noticed that both eIF4A3 and Y14 concentrate around the centrosome at the base of primary cilia in the majority of quiescent mNSCs (Fig. 1a, c, e-h and Supplementary Fig. 1a, b). In contrast, ependymal cells do not show a strong eIF4A3 and Y14 staining around centrioles (Fig. 1b, d, e-h and Supplementary Fig. 1c, d). The reduced concentration of both proteins around centrioles in ependymal cells was not due to an overall lower expression of the two proteins as the nuclear signals of eIF4A 3 and Y14 increased by 1.5 fold in ependymal cells compared to quiescent mNSCs (Supplementary Fig. 1e, f). 
a

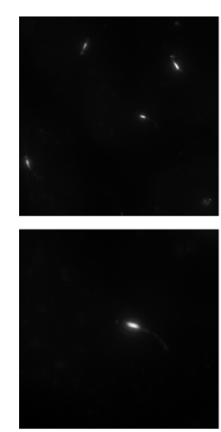

PolyGlu-Tub

b
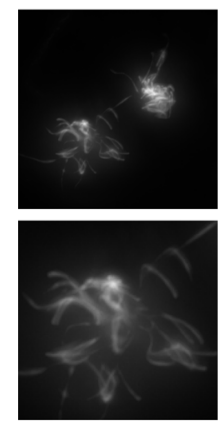

PolyGlu-Tub

c
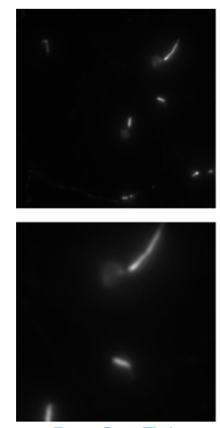

PolyGlu-Tub

d
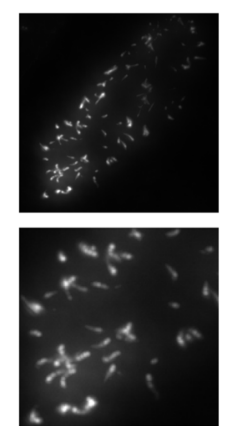

PolyGlu-Tub

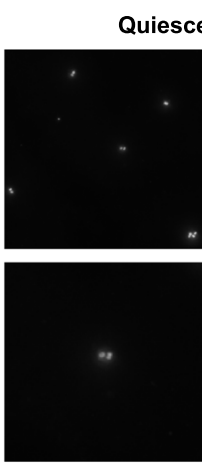

FOP
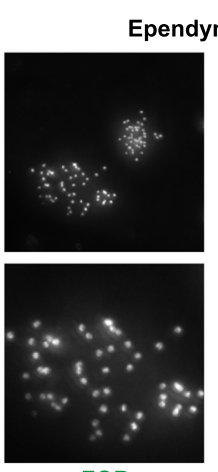

FOP

Quiescent mNSC
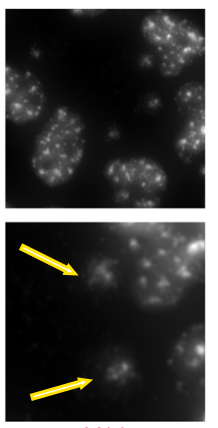

Y14

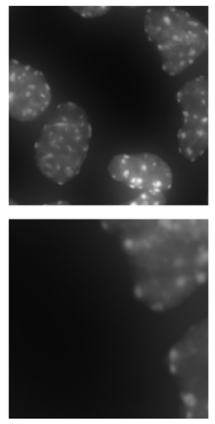

Hoechst

Ependymal cell
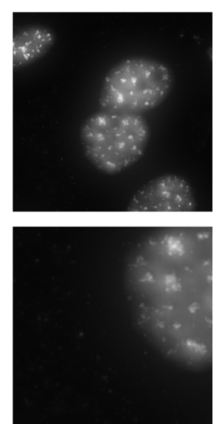

Y14
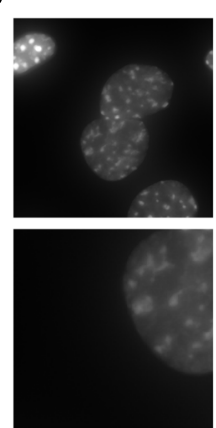

Hoechst

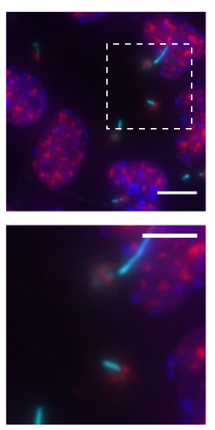

Merge
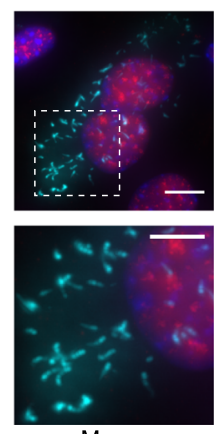

Merge e

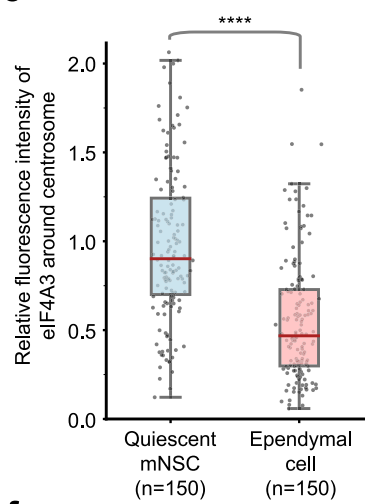

f

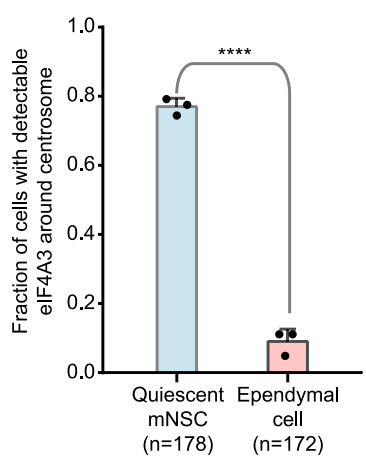

g

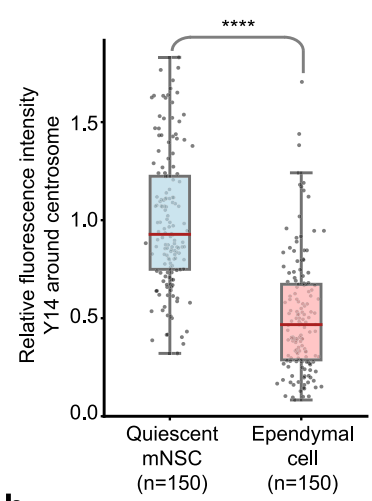

h

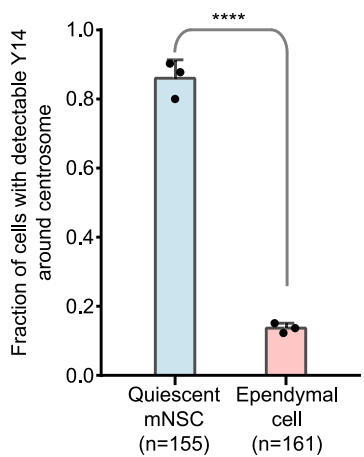

Together, these data showed that at least two EJC core proteins accumulate in the vicinity of centrioles in monociliated mNSCs and this cytoplasmic localization decreases upon differentiation into ependymal cells.
EJC core components accumulate around centrosomes in ciliated quiescent RPE1 cells. To test the generality of this observation, we investigated the localization of EJC core proteins in the telomerase-immortalized RPE1 cell line hTERT-RPE1, a popular 
Fig. 1 EJC core components, elF4A3 and Y14, strongly localize around centrosomes in quiescent mNSC, but not in differentiated ependymal cells. Quiescent mNSC (mouse neural stem cell; $\mathbf{a}, \mathbf{c}$ ) and multiciliated ependymal cells (b, d) were stained for elF4A3 (a, b) or Y14 (c, d). Centrosomes were labeled by FOP antibody. Primary cilia and centriole were stained by poly-glutamylated tubulin (PolyGlu-Tub) antibody. Nuclei were stained by Hoechst. Images result from maximum intensity projections of $12 \mathrm{z}$-stacks acquired at every $0.5 \mu \mathrm{m}$. Lower panels show enlarged images that are marked by white dashed square in the upper panel. Scale bars in the upper and lower panels are 5 and $3 \mu \mathrm{m}$, respectively (a-d). Yellow arrows depict concentrated EJC proteins ( $\mathbf{a}, \mathbf{c})$. Fluorescence intensities for elF4A3 (e) and for Y14 (h) were quantified in $2 \mu \mathrm{m}$ circles around centrosomes and plotted as fluorescence intensities relative to the average fluorescence intensity in quiescent mNSC (set as 1.0). The red lines mark the median values and values between the 25 th lower percentile and 75th higher percentile are in the box. Whiskers above and below the box correspond to 0.35th lower percentile and 99.65th higher percentile, respectively $(\mathbf{e}, \mathbf{g})$. The fraction of cells with detectable centrosomal elF4A3 (f) or Y14 (h) was determined in either quiescent mNSC or ependymal cells. Data are presented by mean + SD of three independent experiments $(\mathbf{f}, \mathbf{h}){ }^{\star \star \star \star} P \leq 0.0001$, two-tailed Mann-Whitney test (e, $\left.\mathbf{g}\right)$ and twotailed $t$-test $(\mathbf{f}, \mathbf{h})$. The number of cells analyzed in three independent experiments is provided $(\mathbf{e}-\mathbf{h})$. Source data are provided as a Source Data file.

cellular model to study primary cilia ${ }^{52,53}$. After 2 days of serum starvation, around $80 \%$ of RPE1 cells possessed a primary cilium, compared to only $9 \%$ in proliferating cells cultivated with serum (Fig. 2a-c). Y14 and MAGOH form a stable and obligated heterodimer ${ }^{54,55}$. Given that no antibodies against MAGOH provided specific immunofluorescence signals, we did not analyze MAGOH localization. And, as previously observed in other cells $^{51,56}$, MLN51 is mainly detected in the cytoplasmic compartment of RPE1 cells (Supplementary Fig. 2e, f). It generated a background preventing the detection of its potential enrichment around centrosomes. As expected, eIF4A3 and Y14 were mainly localized in the nuclear compartment where they concentrated in nuclear speckles, corresponding to punctuate domains enriched in splicing factors and labeled by SC35 and/or 9G8 antibodies (Fig. 2a, b, Supplementary Figs. 1g-j and 2a, b). Remarkably, in a large fraction of quiescent RPE1 cells, eIF4A3 and Y14 also concentrated around centrioles (Fig. 2a, d and Supplementary Fig. 2a, c). In contrast, eIF4A3 and Y14 were not accumulating around the centrosome of proliferating cells (Fig. 2b and Supplementary Fig. 2b). The relative fluorescence intensity of eIF4A3 and Y14 around centrosome was 1.5 times higher in quiescent cells than in proliferating cells (Fig. 2e and Supplementary Fig. 2d). In quiescent RPE1 cells, eIF4A3 and Y14 both accumulate around centrosomes at the base of primary cilia like in quiescent NSC.

We next investigated cell cycle-dependent localization of EJC proteins. For this, we followed eIF4A3 and Y14 signals during a $24 \mathrm{~h}$ time-course triggered by serum addition to quiescent RPE1 cells (Fig. 2f-h and Supplementary Fig. 3a, b). As previously reported $^{57}$, the proportion of monociliated cells decreased following a two-steps mode, with roughly $20 \%$ of ciliated cells left after $24 \mathrm{~h}$ (Fig. $2 \mathrm{~g}$ ). The amount of eIF4A3 and Y14 started to decrease after $8 \mathrm{~h}$ of serum addition and was similar to the amount observed in unsynchronized proliferating cells (Fig. $2 \mathrm{f}, \mathrm{h}$ and Supplementary Fig. 3a, b). As the number of cells in S phase peaked at $16 \mathrm{~h}$ after serum addition, accumulation of EJC proteins around centrosomes most likely declined during the $\mathrm{S}$ phase (Supplementary Fig. 3d, e).

Serum starvation is a kind of stress that can induce translational repression ${ }^{58-61}$. A short sodium arsenite treatment induced the formation of stress granules detected by TIA-1 and eIF4E antibodies (Supplementary Fig. 4a, b). However, EJC accumulation around centrosome did not correspond to stressinduced foci because serum starvation did not lead to accumulation of the stress granule protein TIA-1 in RPE1 cells. We also investigated the impact of translation inhibition by incubating RPE1 cells with either puromycin that dissociates translating ribosomes or cycloheximide that stalls elongating ribosomes onto mRNAs. Both treatments weakly increased the centrosomal accumulation of eIF4A3 and Y14 in quiescent RPE1 cells and had little effect in proliferating cells (Supplementary Fig. 4c-j). Serum addition to quiescent cells stimulates protein synthesis. Indeed, using the SUnSET method ${ }^{62}$, we found that global protein synthesis increases by 3 -fold $8 \mathrm{~h}$ after serum re-addition but decreases afterwards (Supplementary Fig. 3f, g). Given that the amount of EJCs around centrosomes decreases later, it is unlikely that its disappearance from centrosomes is linked to a global increase in protein synthesis following serum addition. Therefore, the concentration of EJC proteins at the base of RPE1 primary cilia does not result from stress or partial translation inhibition triggered by serum starvation.

RNA-dependent accumulation of assembled EJCs around centrosomes. One question raised by these results was whether eIF4A3 and Y14 accumulate around centrosomes independently or not. Dual labeling of eIF4A3 and Y14 showed that they colocalize around centrosomes (Fig. 3a). The relative fluorescence intensities of eIF4A3 and Y14 followed similar patterns when plotted along lines crossing either nuclear speckles where the EJC subunits are concentrated (Fig. 3b), or centrosomes (Fig. 3c). Analysis of 60 individual centrosomes and speckles indicated a very high correlation of localization of the two proteins in both places (Fig. 3d). To further support the hypothesis that eIF4A3 and Y14 co-exist in assembled EJCs near centrosomes in quiescent cells, we down-regulated the expression of either eIF4A3 or Y14 by RNA interference. RT-qPCR, Western blotting and immunofluorescence monitoring showed that silencing of one protein did not affect the expression of the other one (Fig. $3 \mathrm{e}-\mathrm{i}$ and Supplementary Fig. 5a, b). In contrast, down-regulation of Y14 strongly reduced eIF4A3 intensity around centrosomes (Fig. 3h, j), and conversely down-regulation of eIF4A3 strongly reduced Y14 accumulation around centrosomes (Supplementary Fig. 5a, c).

Since EJCs are assembled onto RNA ${ }^{12,51}$, we next investigated whether their presence around centrosomes depends on RNA. Quiescent RPE1 cells were permeabilized, incubated with RNase A before fixation and stained with antibodies. As a positive control, we showed that the number of P-bodies (cytosolic RNP granules involved in mRNA storage ${ }^{63}$ ) was reduced by 4 fold upon such treatment (Supplementary Fig. 5d-f). This short RNaseA treatment slightly reduced the amount of eIF4A3 and Y14 in the vicinity of these foci (Supplementary Fig. 5j, k), as expected given that EJCs are assembled around nuclear speckles ${ }^{51}$. Remarkably, RNase A strongly reduced the amount of both eIF4A3 and Y14 around centrosomes (Fig. 3k, 1 and Supplementary Fig. $5 g-i)$.

Together, the interdependent centrosomal colocalization of eIF4A3 and Y14, and its susceptibility to RNase strongly support that these proteins accumulate around centrosomes of quiescent cells as part of assembled EJC complexes.

Microtubule-dependent transport of centrosomal EJCs. Multiple mechanisms allow the transport and/or the concentration of 
a

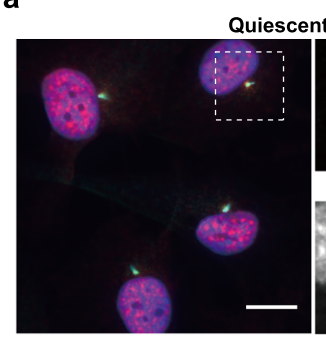

C

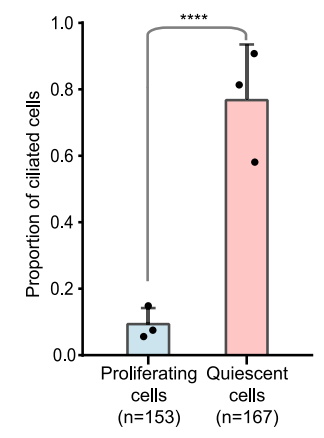

f

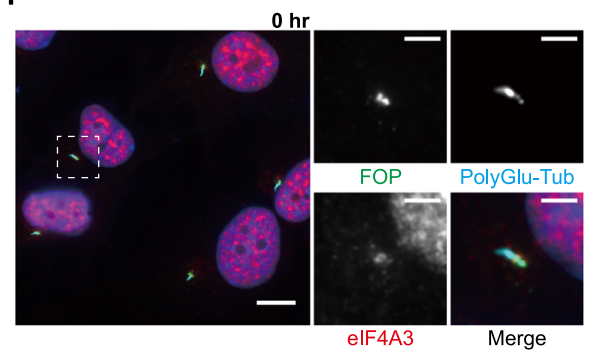

$16 \mathrm{hr}$
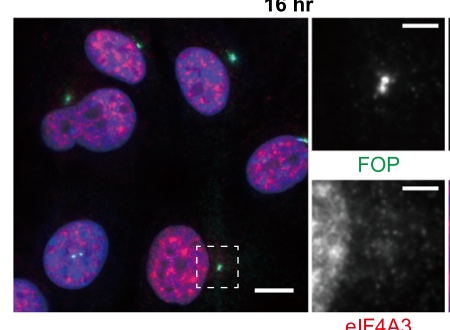

elF4A3

g

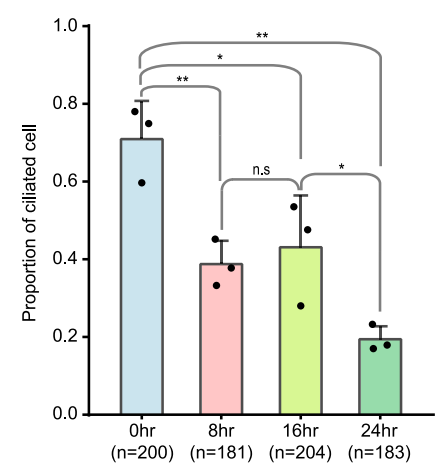

transcripts in specific cellular locations ${ }^{64}$. Active transport of mRNP particles notably use cytoskeleton structures ${ }^{65,66}$. Centrosomes function as the major microtubule-organizing centers ${ }^{42}$. Therefore, we first questioned whether the accumulation of EJCs around centrioles in quiescent cells relies on the microtubule network. When quiescent RPE1 cells were treated with

d b
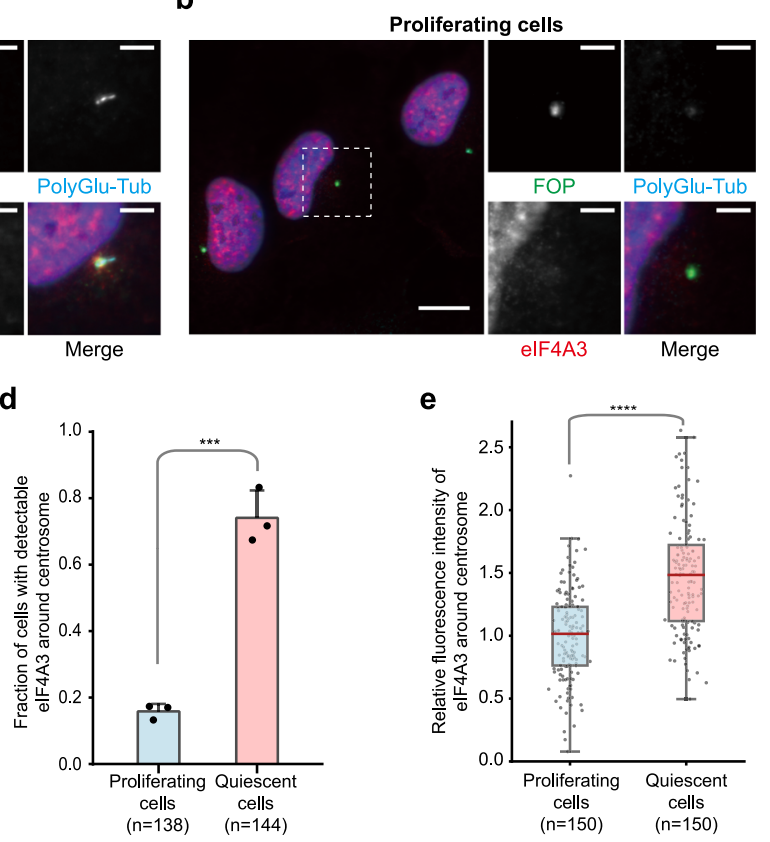

e
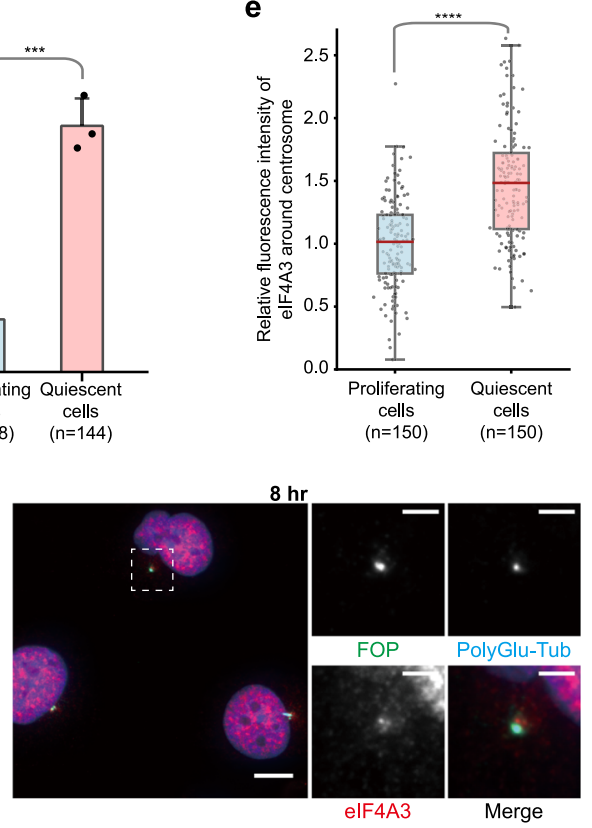

$24 \mathrm{hr}$
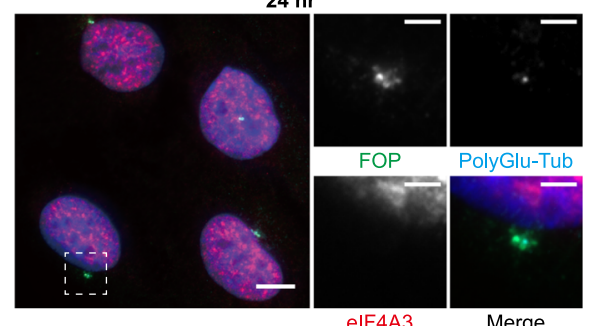

h

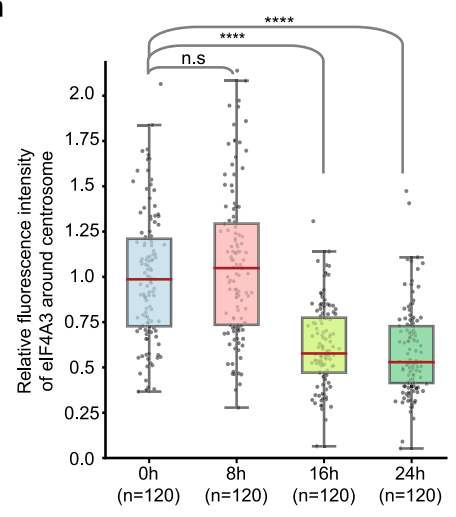

nocodazole for two hours, a well-known microtubule destabilizer, microtubules disappeared (Supplementary Fig. 6a, b). However, poly-glutamylated tubulin immunostaining of both centrioles and cilia was not significantly affected (Fig. 4a). In contrast, this treatment reduced the fluorescence intensities of eIF4A3 and Y14 around centrosomes, by $60 \%$ and $50 \%$, respectively (Fig. $4 \mathrm{a}$, $\mathrm{d}$ and 
Fig. 2 EJC core component accumulates around centrosomes in quiescent RPE1 cells and decreases upon cell cycle re-entry. Proliferating (a) and quiescent (b) RPE1 cells and RPE1 cells incubated with 10\% serum containing media during indicated times after quiescence (f) were stained for elF4A3. Centrosomes were labeled by FOP antibody and primary cilia, and centriole were stained by poly-glutamylated tubulin (PolyGlu-Tub) antibody. Nuclei were stained by Hoechst. Right panels show enlarged images of the white dashed square in the left panel. Scale bars in the left panels are $10 \mu \mathrm{m}$, and scale bars in right panels are $3 \mu \mathrm{m}(\mathbf{a}, \mathbf{b}, \mathbf{f})$. The proportion of ciliated cells was determined in either proliferating or quiescent cell populations (c) and the cell populations incubated with serum containing media during indicated incubation times $(\mathbf{g}$ ). The fraction of cells with detectable elF4A3 (d) was determined in either proliferating or quiescent RPE1 cells. Column graphs present mean + SD of three independent experiments (c, $\mathbf{d}, \mathbf{g}$ ). Quantifications of elF4A3 fluorescence intensities $(\mathbf{e}, \mathbf{h})$ were performed as described in the legend of Fig. 1 except that average fluorescence intensities of elF4A3 in proliferating cells (e) and cells with $\mathrm{O}$ h incubation (h) are set to 1.0. The red lines mark the median values and values between the 25th lower percentile and 75 th higher percentile are in the box. Whiskers above and below the box correspond to 0.35th lower percentile and 99.65th higher percentile, respectively $(\mathbf{e}, \mathbf{h})$. n.s. $P>0.05,{ }^{\star} P \leq 0.05,{ }^{\star \star} P \leq 0.01,{ }^{\star \star \star} P \leq 0.001$, and ${ }^{\star \star \star \star} P \leq 0.0001$, two-tailed Mann-Whitney test (e, h) and two-tailed $t$-test (c, d, $\left.\mathbf{g}\right)$. The number of cells analyzed in three independent experiments is provided $(\mathbf{c}-\mathbf{e}, \mathbf{g}, \mathbf{h})$. Source data are provided as a Source Data file.

Supplementary Fig. 6d, g). These observations indicate that EJCs accumulate around centrosomes in a microtubule-dependent manner. Given that centrosome nucleate the minus ends of microtubules, minus-end directed motors and notably cytoplasmic dynein might transport EJC-bound particles to centrosomes. To test this hypothesis, we incubated quiescent RPE1 cells for $90 \mathrm{~min}$ with Ciliobrevin $\mathrm{D}$, a cell-permeable inhibitor of dynein. Indeed, such treatment efficiently disrupted the Golgi network immunostained with GM130 antibodies, as previously reported $^{67}$ (Supplementary Fig. 6c). Interestingly, the Ciliobrevin $\mathrm{D}$ treatment also reduced the fluorescence intensities of eIF4A3 and Y14 around centrosome by $40 \%$ (Fig. 4b, e and Supplementary Fig. 6e, h) showing that centrosomal EJCs concentration requires dynein motors.

Microtubules form a dynamic network undergoing permanent polymerization and depolymerization. To further investigate the dynamic aspect of EJC transport to centrosomes, we performed microtubule regrowth assays. When quiescent cells were icechilled, the microtubule network labeled with $\beta$-tubulin or $\alpha$ tubulin antibodies almost completely disappeared, and the amounts of centrosomal eIF4A3 and Y14 were reduced by 2 fold (Fig. 4c, f and Supplementary Fig. 6f, i). Placing cells back at $37^{\circ} \mathrm{C}$ induced microtubule regrowth. Already one minute after addition of $37^{\circ} \mathrm{C}$ media, astral structures reappeared at microtubule organizing centers and $15 \mathrm{~min}$ later, the microtubule network was almost completely reconstituted (Fig. 4c and Supplementary Fig. 6f). Remarkably, the intensity of eIF4A3 and Y14 at centrosome already increased after one minute back at $37^{\circ} \mathrm{C}$ and reached almost initial levels after $15 \mathrm{~min}$ (Fig. 4c, f and Supplementary Fig. 6f, i).

Together, these data indicated that an important proportion of EJC complexes are rapidly transported to centrosomes of quiescent RPE1 in a microtubule- and dynein-dependent manner.

Basal body localization of NIN mRNAs but not BICD2 mRNAs is both EJC- and translation-dependent. Finding EJCs assembled on RNA prompted us to search for transcripts localized around centrosomes in ciliated RPE1 cells. We used a high-throughput smFISH strategy (schematized in Fig. 5a; see also Safieddine et al. ${ }^{68}$ ) to screen about 700 mRNAs encoding centrosome-related and cilium-related proteins (Supplementary Data 1). Briefly, we generated 50-100 distinct single-stranded RNA probes for each mRNA. The probes were flanked by two overhangs that hybridize with fluorescently labeled locked nucleic acids (LNA). The probe mixtures were hybridized on fixed cells following a smiFISH procedure as described previously ${ }^{69}$. We used RPE1 cells stably expressing centrin1-GFP for centrosome labeling and antibodies against Arl13b to stain primary cilia (Fig. 5a and Supplementary Fig. 7a). Among the different mRNAs investigated, we found 21 mRNAs that exhibit non-random intracellular distribution (Supplementary Data 1). For example, CHD3 (Chromodomain Helicase DNA binding protein 3; a component of NuRD chromatin remodeling complex ${ }^{70}$ ) accumulated in cytoplasmic protrusions while NEK9 mRNA (also known as Nerccl; serine/threonine kinase controlling centrosome separation during prophase ${ }^{71}$ ) was distributed randomly throughout the cytoplasm (Supplementary Fig. 7a). Remarkably, two mRNAs, BICD2 and NIN, specifically concentrated around centrosomes at the base of cilia (Supplementary Fig. 7a). Bicaudal D2 (BICD2) is a dynein adaptor involved in RNP particles and vesicles trafficking along microtubule network ${ }^{72}$. Ninein (NIN) is a core component of centrosomes required for microtubule nucleation and anchoring to centrosome $\mathrm{e}^{73,74}$. While we screened a large fraction of mRNAs corresponding to the centrosomal and cilium proteomes, the screen was not exhaustive. Hence, additional mRNAs might localize there (see the section "Discussion").

Next, to determine whether BICD2 and NIN transcripts were associated to EJCs, we performed RNA immunoprecipitations. RT-qPCR showed that both transcripts were efficiently and specifically precipitated with eIF4A3 and Y14 antibodies but not with antibodies against the unrelated protein Rab5 (Supplementary Fig. 8a). This enrichment was specific because the intron-less SFM3B5 and SDHAF1 transcripts were not precipitated under the same conditions (Supplementary Fig. 8a). Therefore, a significant proportion of BICD2 and NIN mRNAs are bound to EJCs in quiescent RPE1 cells.

We next tested whether the localization of BICD2 and NIN mRNAs was EJC-dependent. Neither eIF4A3 nor Y14 knockdown affected the centrosomal localization of BICD2 mRNA (Fig. 5b, d). In contrast, both knock-downs strongly perturbed NIN mRNA localization that became more dispersed (Fig. 5c, e). Measurement of NIN mRNAs expression by RT-qPCR showed that EJC knock-downs did not alter its overall expression (Supplementary Fig. 8b). Therefore, EJCs actively participate to the centrosomal localization of NIN transcripts.

We and others previously observed that the localization of PCNT and ASPM mRNAs to centrosome during early mitosis is translation-dependent ${ }^{75,76}$. Although cycloheximide treatment had no effect, puromycin treatment prevented the accumulation of both NIN and BICD2 mRNAs around centrosomes in quiescent RPE1 cells (Supplementary Fig. 7b-e).

Taken together, our data suggest that EJCs contribute to the transport and localization at centrosomes of NIN transcripts undergoing translation, whereas the localization of BICD2 mRNA only requires translating ribosome.

EJC protein depletion impairs both centrosome organization and ciliogenesis. Ninein (NIN) is a core component of centrosomes located at the proximal end of each centriole and at subdistal appendages of mother centrioles. It contributes to microtubule nucleation and anchoring to centrosomes ${ }^{73,74}$. We investigated the NIN protein by immunofluorescence. We found that either eIF4A3 or Y14 knock-down reduced by half the amount of 
a
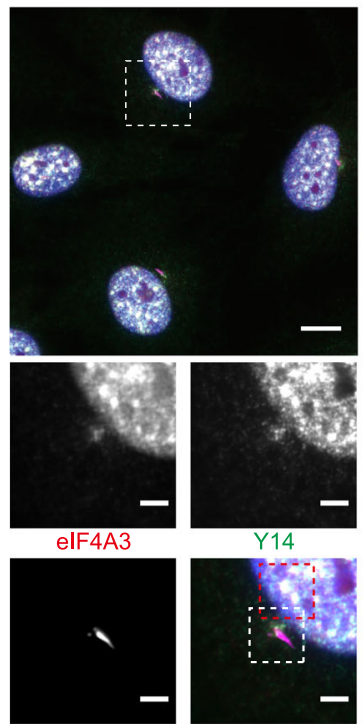

PolyGlu-Tub b

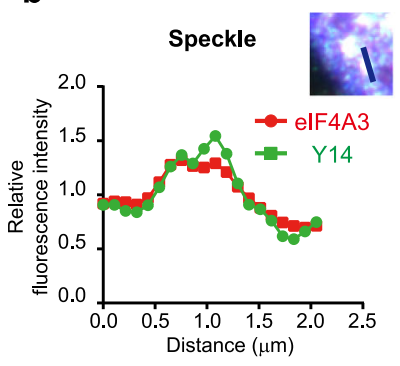

C

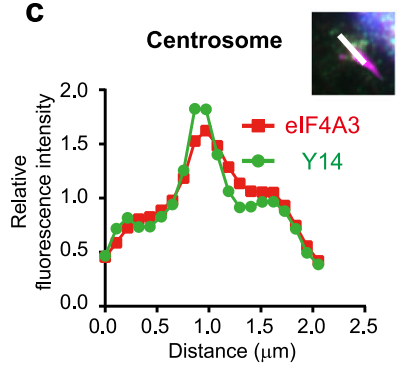

d

e

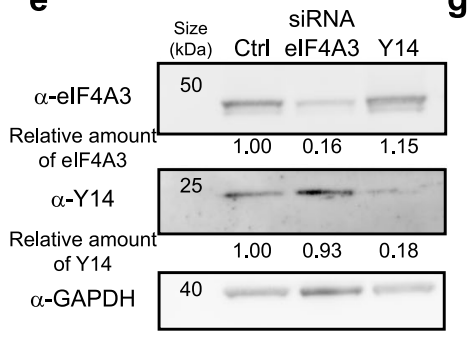

i

h

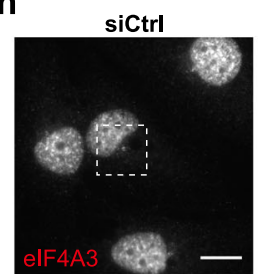

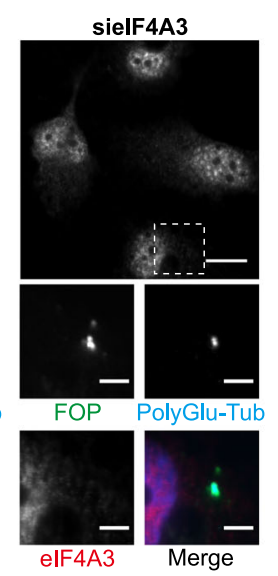

elF4A3 Merge

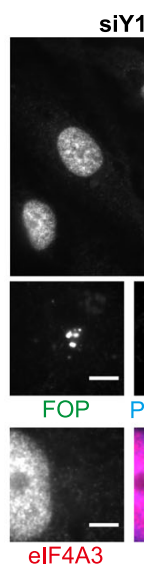

elF4A3
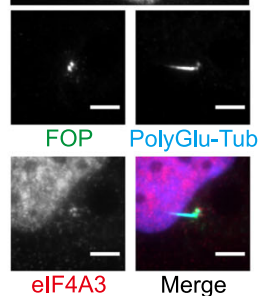

elF4A3 Merge

siY14

k

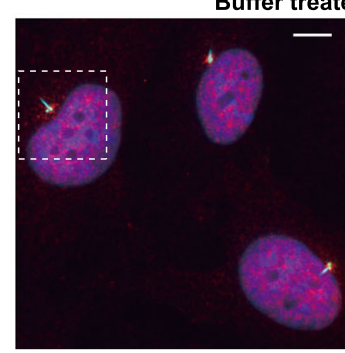

Buffer treated condition

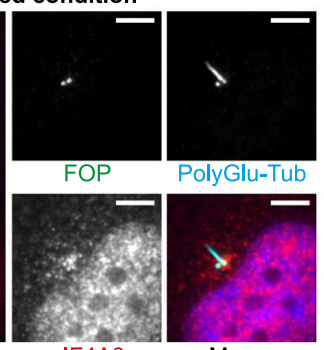

elF4A3

Merge

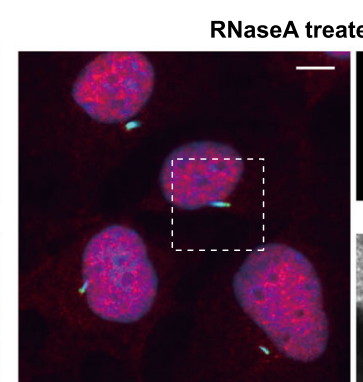

RNaseA treated condition

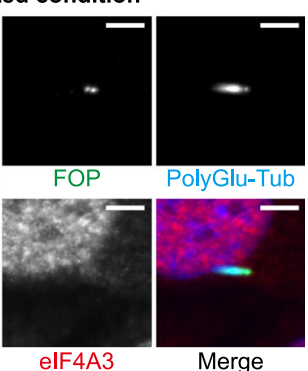

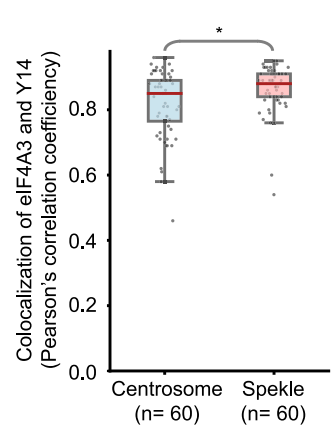

f

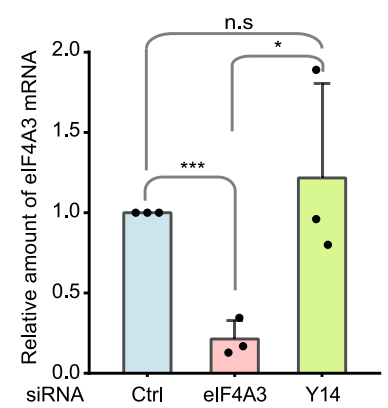

g
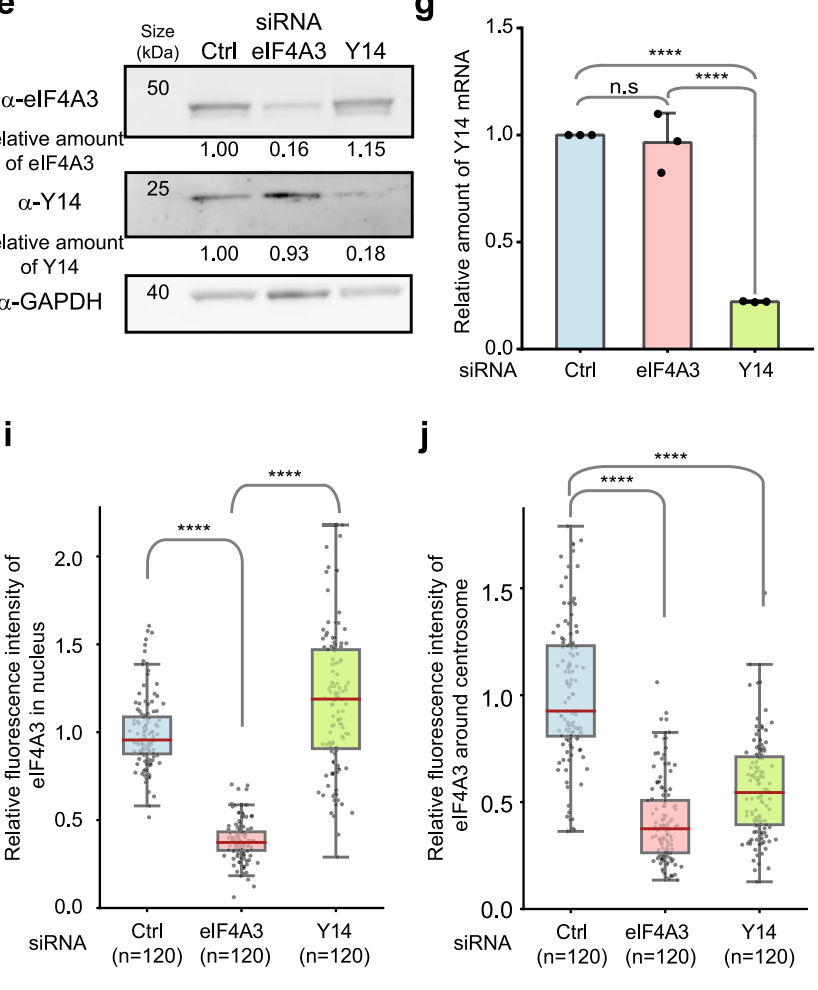

j

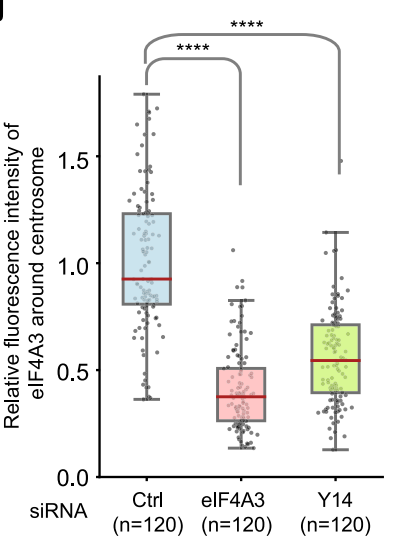

NIN protein detected around centrosomes (Fig. 6a, b). This observation prompted us to analyze other centrosomal components. We observed that knock-down of either eIF4A3 or Y14 also had a strong effect on PCM-1 and FOP localizations in quiescent RPE1 cells (Fig. 6c-e). In control cells, PCM-1 labeling showed that the PCM and centriolar satellites were mainly concentrated around centrioles and radially distributed from the centrosome in a punctuated manner. eIF4A3 knock-down led to the appearance of PCM-1 dots a few microns away from centrioles, with a dispersed and scattered pattern. A Y14 knock-down had the same impact though less pronounced. In control cells,
FOP staining was very focused although an extended punctuated staining is seen in $15 \%$ of the cells. However, such an extended punctuated FOP staining was observed in $80 \%$ of the cells after knock-down of either eIF4A3 or Y14 (Fig. 6c, d). The specific role of EJC in centrosome organization was further supported by the fact that down regulation of MAGOH showed similar effects (Supplementary Fig. 9). In contrast, the down-regulation of MLN51 showed no impact suggesting that EJC-related functions of MLN51 are most likely transcript and/or cell-specific, as previously reported 38,77 . Thus, EJC depletion triggers defects in centriolar satellite transport. 
Fig. 3 Assembled ECJs on RNA accumulate around centrosomes. Quiescent RPE1 cells were immunolabeled by elF4A3 and Y14 (a). Quiescent cells transfected with indicated siRNAs (h) and permeabilized quiescent cells incubated with RNAse A or not (k) were stained for elF4A3. poly-glutamylated tubulin (PolyGlu-Tub) antibody stains primary cilia and centrioles ( $\mathbf{a}, \mathbf{h}, \mathbf{k})$ and FOP antibody labels centrosomes (h, $\mathbf{k}$ ). Nuclei were stained by Hoechst. Lower $(\mathbf{a}, \mathbf{h})$ or right panels $(\mathbf{k})$ show enlarged images of the white dashed square in upper $(\mathbf{a}, \mathbf{h})$ or left panel $(\mathbf{k})$. $\mathbf{b}$ and $\mathbf{c}$ are enlarged images from red and yellow dashed squares in lower panel (a). Scale bars in upper $(\mathbf{a}, \mathbf{h})$ or left $(\mathbf{k})$ panels and lower $(\mathbf{a}, \mathbf{h})$ or right panels $(\mathbf{k})$ panels are 10 and $3 \mu \mathrm{m}$, respectively. Relative fluorescence intensity of eIF4A3 and Y14 along the lines were plotted (b, $\mathbf{c}$ ). Average fluorescence intensity on the line is set to 1.0 . Colocalization of elF4A3 and Y14 was analyzed in $2 \mu \mathrm{m}$ circles around centrosomes and nuclear speckles and plotted (d, perfect colocalization is set to 1.0). siRNAs were validated by either Western blotting or RT-qPCR. Relative protein (e) or RNA level (f, $\mathbf{g}$ ) normalized by GAPDH is depicted (average values of siCtrl is set to 1.0). Relative fluorescence intensities of elF4A3 in the nucleus (i) and those around centrosome (j, $\mathbf{I})$ were measured as described in Fig. 1 legend [the average fluorescence intensity of elF4A3 in $\operatorname{siCtrl}(\mathbf{i}, \mathbf{j})$ or buffer $(\mathbf{I})$ condition is set to 1.0]. Columns and bars depict mean \pm SD of three independent experiments $(\mathbf{f}, \mathbf{g})$. Boxes represent values between the 25 th lower and 75 th higher percentile, and the red lines mark the median. Whiskers above and below correspond to 0.35 th lower and 99.65th higher percentile, respectively $(\mathbf{d}, \mathbf{i}, \mathbf{j}, \mathbf{I})$. n.s $P>0.05,{ }^{\star} P \leq 0.05$, ${ }^{\star \star \star} P \leq 0.001$, and ${ }^{\star \star \star \star *} P \leq$ 0.0001 , two tailed Mann-Whitney test $(\mathbf{d}, \mathbf{i}, \mathbf{j}, \mathbf{I})$ and two-tailed t-test $(\mathbf{f}, \mathbf{g})$. The number of cells analyzed in three independent experiments is provided $(\mathbf{d}, \mathbf{i}, \mathbf{j}, \mathbf{I})$. Source data are available in a Source Data file.

We also observed that eIF4A3 or Y14 knock-downs reduced both the $\gamma$-tubulin and PCNT fluorescence intensities at centrosomes (Supplementary Fig. 8c-e), in agreement with the fact that NIN and PCM-1 are important for deposition of $\gamma$ tubulin and PCNT on centrosome, respectively ${ }^{73,78}$. However, although EJC knock-down impairs the localization of some centrosome components, it did not induce major changes in the microtubule network revealed by $\beta$-tubulin labeling (Supplementary Fig. 8f).

Ciliogenesis is linked to basal body integrity. As knock-down of either eIF4A3, Y14 or MAGOH decreased the number of ciliated cells by more than $50 \%$ (Fig. 6c, e and Supplementary Fig. 9f, h), an imbalance in EJC dosage in quiescent RPE1 cells strongly impairs both the organization of centrosomes and ciliogenesis.

\section{Discussion}

Here, we show the accumulation of EJC core proteins around basal bodies, which are formed by centrioles at the base of cilia. This was observed both in primary mNSCs and quiescent RPE1 cells. These EJC proteins are assembled on RNA and localized in a microtubule-dependent manner revealing the enrichment of untranslated or partially translated EJC-bound transcripts at centrosomes. A large smFISH screen identifies BICD2 and NIN mRNAs near the base of primary cilia in quiescent RPE1 cells. Knock-down of any EJC core protein prevents NIN mRNAs transport but not BICD2 mRNAs transport. Thus, the EJC plays a crucial role for the spatial enrichment of specific mRNAs at a specific location in human cells. In addition, we provide evidences that an EJC imbalance affects the centrosomal accumulation of NIN protein and other structural components, such as pericentrin and PCM1. Thus, the EJC is associated to defects in centrosomal organization and ciliogenesis.

We provide several complementary evidences that assembled EJCs accumulate in a RNA-dependent manner around basal bodies. EJCs are deposited by the nuclear-splicing machinery and remain stably bound to transcripts until their translation in the cytoplasm $^{15,17}$. So far, there is no evidence that a splicingindependent EJC assembly may occur. Therefore, the RNAdependent enrichment of EJCs at the centrosomes signals the local concentration of spliced transcripts both in mNSC and in human RPE1. Previous studies reported the presence of RNAs in the centrosomal area in different organisms including Tetrahymena pyriformis, Paramecium tetraurelia, Spisula solidissima, Ilyanassa obsolete, Dano rerio, Xenopus laevis, and Drosophila melanogaster ${ }^{75,79-82}$. More recently, four transcripts encoding the central PCM component, pericentrin (PCNT), abnormal spindlelike microcephaly associated protein (ASPM), the nuclear mitotic apparatus protein 1 (NUMA1), and the hyaluronan-mediated motility receptor (HMMR) were detected by single molecule approaches around centrosome of $\mathrm{HeLa}$ cells during cell division $68,75,76$. Here, we identified BICD2 and NIN transcripts as two additional mRNAs concentrated around centrosome at the base of cilia in quiescent RPE1 cells. BICD2, an activating adaptor of dynein, participates to the traffic of both Golgi vesicles and RNP particles along microtubules ${ }^{72}$. Ninein, localized to the proximal end of both mother and daughter centrioles, is a core component of subdistal appendages of mother centrioles ${ }^{74}$. Ninein is important for both microtubule anchoring and nucleation at the centrosome $\mathrm{e}^{73}$. The presence of several transcripts around centrosomes at different stages of the cell cycle $^{75,76}$, the detection of centrosomal EJCs in proliferating cells and their accumulation during quiescence are all echoes of a major, spatially restricted and dynamic post-transcriptional program crucial for the centrosome functions, both during cell division and cilia formation.

Various mechanisms can lead to mRNP enrichment at particular subcellular locations, such as an active transport along cytoskeletal tracks, passive diffusion coupled to site-specific anchoring or local protection from degradation $64,65,83$. It has long been considered that most localized mRNPs are transported in a translationally repressed state to prevent ectopic expression of the encoded protein and/or favor the assembly of protein complexes. However, there are growing evidences of widespread co-translational transports ${ }^{84,85}$. Recently, a large dual protein-mRNA screen in human cells revealed that the majority of the transcripts displaying specific cytoplasmic locations reach their destination in a translation-dependent manner ${ }^{76}$. Cotranslational mRNA transport is notably essential for the targeting of membrane and secreted proteins to the endoplasmic reticulum. In this case, the cytosolic translation of transcripts is arrested after translation of a signal sequence that mediates the transport of the ribosome-bound mRNP to the endoplasmic reticulum where translation resumes after translocation of the nascent polypeptide ${ }^{86}$. The delivery of PCNT and ASPM mRNAs to centrosomes requires active polysomes as well as microtubules and dynein activity ${ }^{75,76}$, and direct visualization of single polysomes in live cells with the SunTag showed that the ASPM and NUMA1 polysomes are actively transported to mitotic centrosomes ${ }^{68}$. Recently, a large dual protein-mRNA screen in human cells revealed that the majority of the transcripts displaying specific cytoplasmic locations reach their destination in a translationdependent manner ${ }^{76}$. Here, we complete this list by showing that the accumulation NIN and BICD2 mRNA around centrosome at the base of primary cilia is highly sensitive to puromycin treatment but not to cycloheximide treatment (Supplementary Fig. S7), strongly suggesting that nascent peptides are necessary for correct targeting. BICD2 and NIN proteins are direct partners of dynein ${ }^{87}$ and the $\mathrm{N}$-terminal region of Ninein is important for 
a

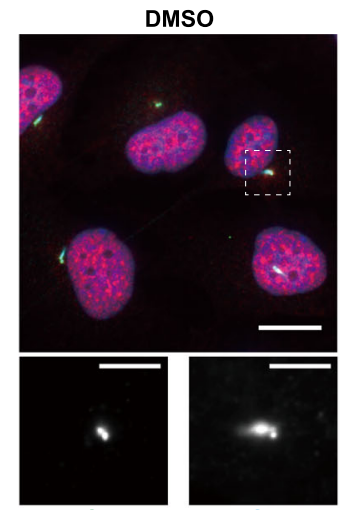

FOP

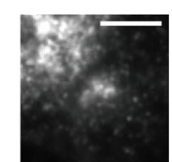

elF4A3

PolyGlu-Tub

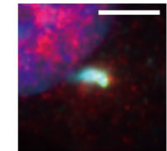

Merge

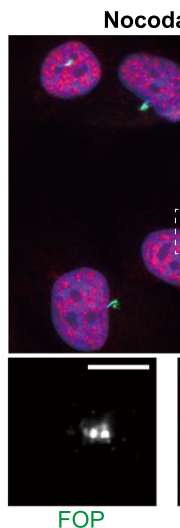

Nocodazole

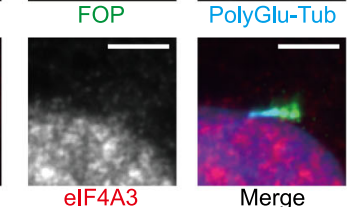

C
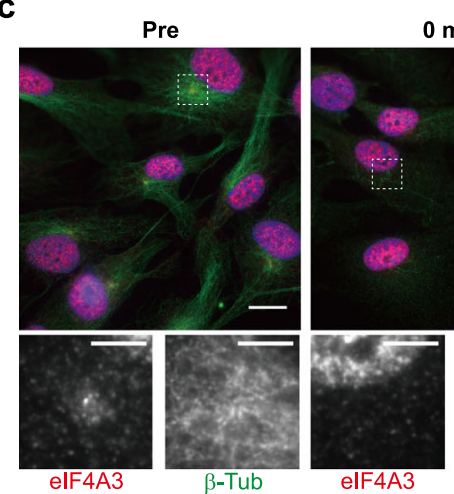

0 min

$1 \mathrm{~min}$

b
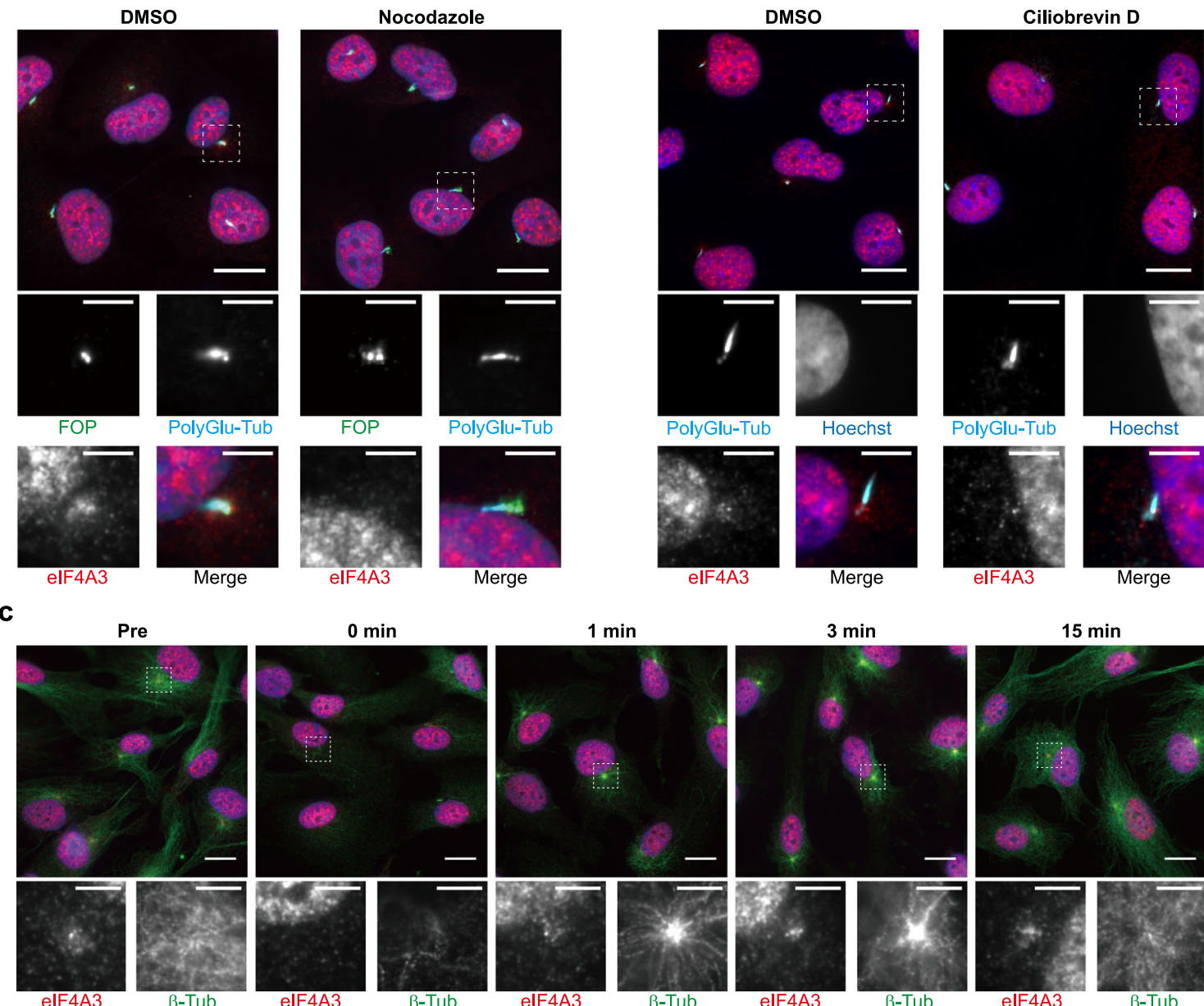

elF4A3
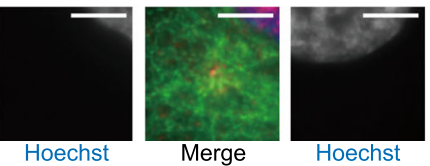

Hoechst

$\mathbf{e}$

d
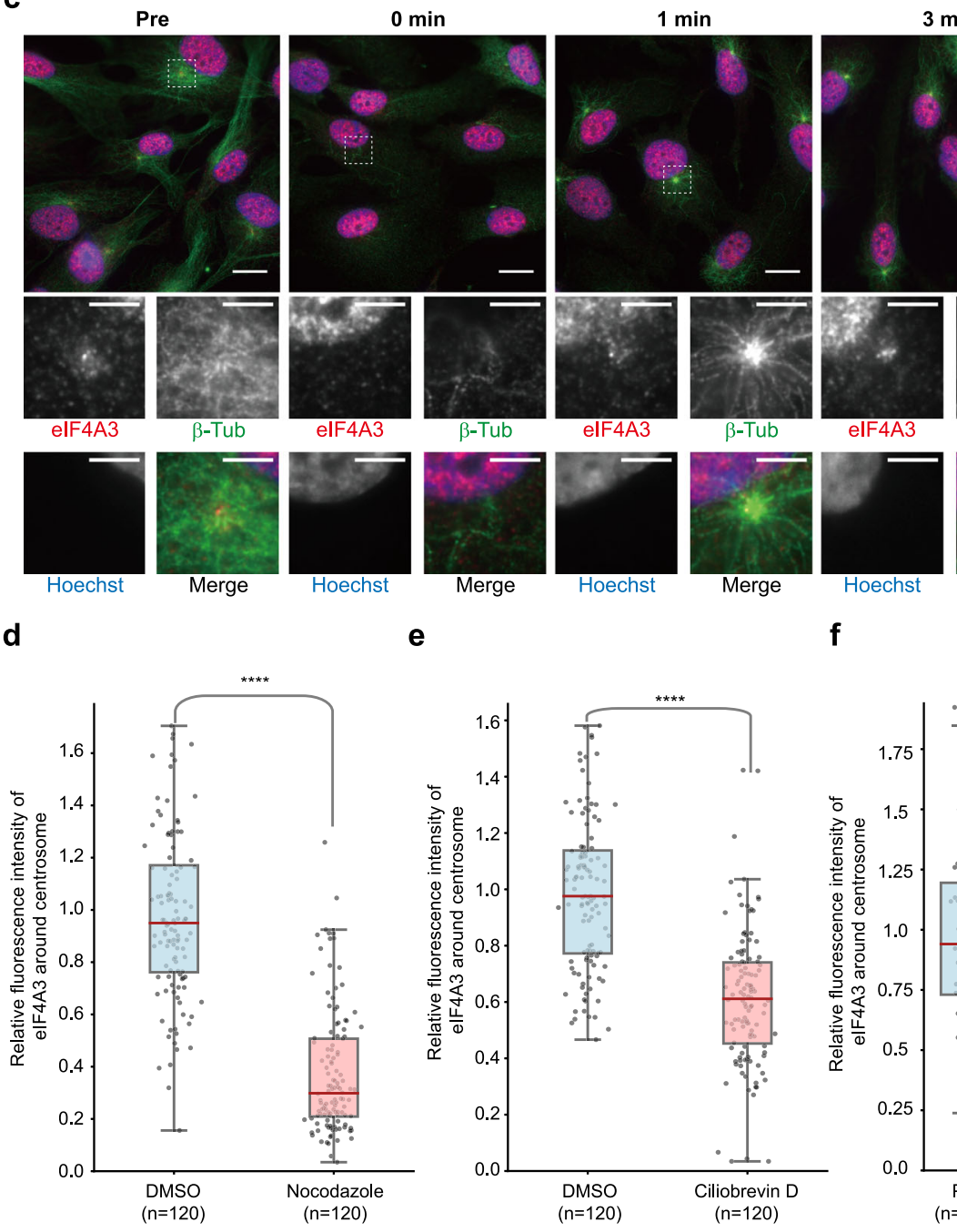

$\min$
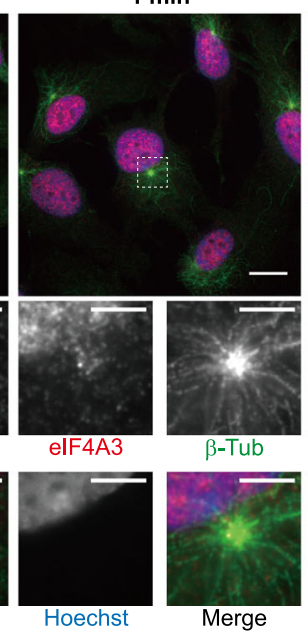

Hoechst

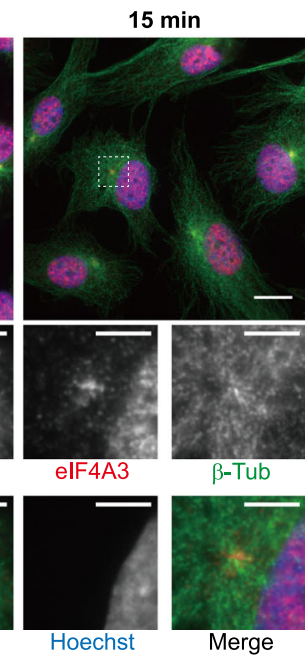

$\mathbf{f}$

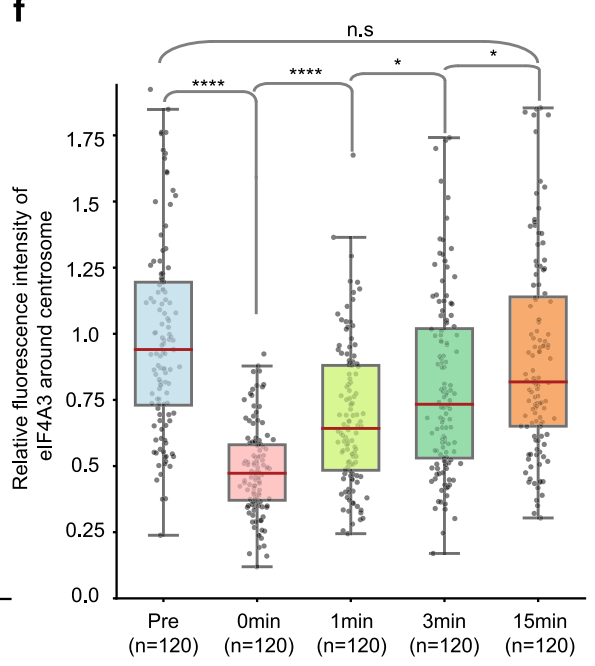

protein targeting to mother centrioles ${ }^{73}$. It is tempting to speculate that BICD2 and NIN nascent peptides somehow contribute to the co-translational and dynein-dependent delivery of their transcripts to achieve protein synthesis at their final destination.

So far, only one example of transcript localization requiring the EJC is known. It is the oskar mRNA that is transported from nurse cells to the posterior pole of D. melanogaster oocytes 88,89 Here, we report a second EJC-dependent localized transcript, the NIN mRNA. It is first described in mammals, revealing that this phenomenon is not an exception restricted to fly. The EJC is likely involved in the subcellular localization of other transcripts yet to be identified. Multiple cis-acting and trans-acting factors 
Fig. 4 An active microtubule-dependent transport is required to maintain EJC localization around centrosomes. elF4A3 antibody-stained quiescent RPE1 cells treated with either DMSO or Nocodazole (a), either DMSO or CiliobrevinD (b), and chilled quiescent cells subjected to a microtubule regrowth assay (c). Centrosomes were labeled by FOP antibody and primary cilia and centriole were stained by poly-glutamylated tubulin (PolyGlu-Tub) antibody $(\mathbf{a}, \mathbf{b})$. Microtubules were stained by $ß$-tubulin ( $ß$-tub in figure) antibody (c). Nuclei were stained by Hoechst. Lower panels show enlarged images marked by white dashed square in the upper panel. Scale bars in the upper and lower panels are 10 and $3 \mu \mathrm{m}$, respectively. Quantification of fluorescence intensities of elF4A3 (d-f) were performed as described in the legend of Fig. 1. The average fluorescence intensities for elF4A3 in DMSO treated cells (d, e) or in pre-incubated quiescent cells (f) are set to 1.0. Boxes represent values between the 25th lower and 75th higher percentile, and the red lines mark the median. Whiskers above and below correspond to 0.35 th lower and 99.65 th higher percentile, respectively. n.s. $P>0.05,{ }^{\star} P \leq 0.05$, and ${ }^{\star \star \star \star} P \leq 0.0001$, two tailed Mann-Whitney test. The number of cells analyzed in three independent experiments is provided (d-f). Pre designates pre-incubation. Source data are provided as a Source Data file.

participate to the active transport and the translational repression of oskar mRNP before it reaches the posterior pole of embryo where the protein Osk is produced ${ }^{64,88,89}$. In this multistep pathway, the EJC is only one of the actors and its precise role remains unclear. In contrast to oskar mRNA, the localization of NIN mRNA requires ongoing translation in addition to the EJC. The combination of these two signals is at first surprising because EJCs deposited on the mRNA ORF are expected to be disassembled by scanning ribosomes. Ribosomes might be halted before reaching the end of the NIN mRNA ORF. The differential sensitivity of NIN mRNA localization to cycloheximide and puromycin, suggests that a nascent NIN polypeptide bound to halted ribosomes cooperates with downstream EJCs for NIN mRNA targeting to centrosome where translation would resume. It is also worth noting that NIN mRNA is $10 \mathrm{~kb}$ long and has 30 introns, thus possibly requiring a particular EJC-driven packaging for transport. Although the molecular mechanisms involved remain unclear, the differential EJC-requirement for BICD2 and NIN mRNAs localization indicate that different pathways orchestrate the ballet of transcripts accumulating around centrosome during the different phases of the cell cycle $\mathrm{e}^{75,76}$ (Fig. 7).

In quiescent cells, the oldest centriole of the centrosome converts into a basal body that nucleates a non-motile primary cilium. This organelle serves as a cellular antenna and constitutes a signaling hub for both chemical and mechanical external stimuli leading to cell-fate decisions such as cell cycle re-entry or cell differentiation ${ }^{90}$. In the brain, primary cilia sense signaling molecules present in the cerebral spinal fluid ${ }^{91}$. Untranslated and partially translated mRNAs unmasked by EJCs and parked around the basal body might wait for external signals to synthesize their protein products on request and contribute to centrosome organization, ciliogenesis, and cilia functions. Here, we show that downregulation of EJC core factors in RPE1 cells affects the localization of NIN mRNAs and consequently, the amount of NIN protein at centrosomes (Fig. 6). Furthermore, it leads to centriolar satellite scattering, pericentriolar assembly/composition defects and to abnormal ciliogenesis (Fig. 6 and Supplementary Figs. 8, 9). Centriolar satellites are dynamic granules transported towards centrosomes along microtubules by a dynein-dependent mechanism ${ }^{92}$ that are essential for centrosome assembly as well as ciliogenesis $^{78,93}$. Therefore, the targeting of EJC-bound transcripts such as NIN mRNA toward centrosomes and possibly their local translation is critical for centrosome structure and cilia formation.

Brain development is particularly susceptible to centrosome dysfunction and defects in several centrosome components are associated with microcephaly 94,95 . Interestingly, mouse haploinsufficiencies in EJC core factors are all associated with defects in neural stem cell division ${ }^{29,37}$. A reduction in IP and an increased apoptosis of progeny are observed and result in neurogenesis defaults and ultimately microcephaly. In addition, a deficient Ninein expression in embryonic mouse brain causes premature depletion of progenitors ${ }^{96}$. Thus, it is tempting to speculate that
EJC-linked neurodevelopmental abnormalities observed in mouse models as well as in human syndromes at least in part originate from centrosomal and primary cilia dysfunctions in NSC, triggered by a defective post-transcriptional EJC-dependent gene regulation.

\section{Methods}

Animals. All animal studies and ethical regulations were performed in accordance with the guidelines of the European Community and French Ministry of Agriculture and were approved by the Direction départementale de la protection des populations de Paris (Approval number APAFIS\#9343-201702211706561 v7). The mice used in this study have already been described and include: RjOrl:SWISS (Janvier Laboratories).

Primary ependymal cell cultures and differentiation. mNSC and ependymal cells were prepared following previous reports ${ }^{43,44}$. Newborn mice (P0-P2) were killed by decapitation. The brains were dissected in Hank's solution [10\% Hanks balanced salt solution (GIBCO), 5\% HEPES (GIBCO), 5\% sodium bicarbonate (GIBCO), 1\% penicillin/streptomycin (P/S) (GIBCO)], and the extracted ventricular walls were manually cut into pieces. The telencephalon was incubated in enzymatic digestion solution [DMEM glutamax, 2.8\% (v/v) papain (Worthington 3126), $1.4 \%(\mathrm{v} / \mathrm{v})$ of $10 \mathrm{mg} / \mathrm{ml}$ DNase I, $2.25 \%(\mathrm{v} / \mathrm{v})$ of $12 \mathrm{mg} / \mathrm{ml}$ cysteine] for 45 min at $37{ }^{\circ} \mathrm{C}$ in a humidified $5 \% \mathrm{CO}_{2}$ incubator. Digestion was inactivated by addition of trypsin inhibitors [Leibovitz Medium L15 (GIBCO), $50 \mu \mathrm{g} / \mathrm{ml} \mathrm{BSA}$, $1 \mathrm{mg} / \mathrm{ml}$ trypsin inhibitor (Worthington), $2 \%$ (v/v) $10 \mathrm{mg} / \mathrm{ml}$ DNase I (Worthington)]. Cells were washed with L15 medium and resuspended by DMEM glutamax supplemented with $10 \%$ fetal bovine serum (FBS) and $1 \% \mathrm{P} / \mathrm{S}$. Ependymal progenitors proliferated until cells are confluent (4-5 days) in a poly-L-lysine (PLL)-coated flask. Then, cells were shacked $(250 \mathrm{rpm})$ at RT overnight before treatment with trypsin-EDTA. Then, $1.5 \times 10^{5}-2 \times 10^{5}$ cells were plated on the PLL-coated coverslip and cultivated in DMEM glutamax $10 \%$ FBS, $1 \% \mathrm{P} / \mathrm{S}$. The next day, medium was replaced by serum-free DMEM glutamax $1 \% \mathrm{P} / \mathrm{S}$, to trigger ependymal differentiation gradually in vitro (DIV 0). Cells were fixed with $4 \%$ paraformaldehyde at DIV 1 day and DIV 6 day for quiescent mNSC and ependymal cells, respectively.

RPE1 cell culture and modulations. RPE1 cells were cultivated in DMEM-F12 1:1 (Invitrogen) supplemented with $10 \%$ of fetal bovine serum (FBS, PAN ${ }^{\mathrm{TM}} \mathrm{BIO}$ TECH), and $1 \%$ penicillin and streptomycin. To induce quiescence, RPE1 cells were washed twice with DPBS and incubated for $48 \mathrm{~h}$ with serum-free DMEM F12 ${ }^{97}$. To repress protein synthesis in quiescent RPE1 cell, $100 \mu \mathrm{g} / \mathrm{ml}$ of cycloheximide (TOKU-E) and $300 \mu \mathrm{M}$ of puromycin (InVivoGen) were added for $2 \mathrm{~h}$ and $100 \mu \mathrm{g} / \mathrm{ml}$ of puromycin and $200 \mu \mathrm{g} / \mathrm{ml}$ of cycloheximide were treated for $6 \mathrm{~h}$ to proliferating RPE1 cell before fixation. $100 \mu \mathrm{g} / \mathrm{ml}$ of puromycin and $200 \mu \mathrm{g} / \mathrm{ml}$ of cycloheximide were added for $20 \mathrm{~min}$ before smiFISH. To disrupt microtubules, $3 \mu \mathrm{g} / \mathrm{ml}$ of nocodazole (Sigma) in DMSO was added to the cells. To inhibit dynein protein, $50 \mu \mathrm{M}$ of ciliobrevin D (Calbiochem) was incubated for $90 \mathrm{~min}$. Stress granules were induced by incubating RPE1 cells with $0.5 \mathrm{mM}$ of sodium arsenite (Sigma) for $30 \mathrm{~min}$. To prepare immunofluorescence sample, $5 \times 10^{4}$ of RPE1 cells were plated on PLL-coated coverslips (VWR) one day before quiescence induction and $3 \times 10^{4}$ cells were plated for proliferating condition.

Microtubule regrowth assay. To destabilize the microtubule structure in the cell, quiescent RPE1 cells were left on ice for $30 \mathrm{~min}$. The cold medium was next replaced with pre-warmed medium and the cells were incubated in $37^{\circ} \mathrm{C}$. After indicated incubation times, cells were washed in PBS and fixed.

siRNA transfection. RPE1 cells plated on day 0 were transfected on day 1 with control siRNAs (5'-UGAAUUAGAUGGCGAUGUU-3'), eIF4A3 siRNA (5'-AG ACAUGACUAAAGUGGAA-3'), Y14 siRNA (5'-GGGUAUACUCUAGUUGAA UUUCAUAUUCAACUAGAG-3'), MAGOH siRNA (5'-CGGGAAGTTAAGAT ATGCCAA-3'), and MLN51 siRNA (5'- GAUCGGAAGAAUCCAGCAU-3', $5^{\prime}$ - 


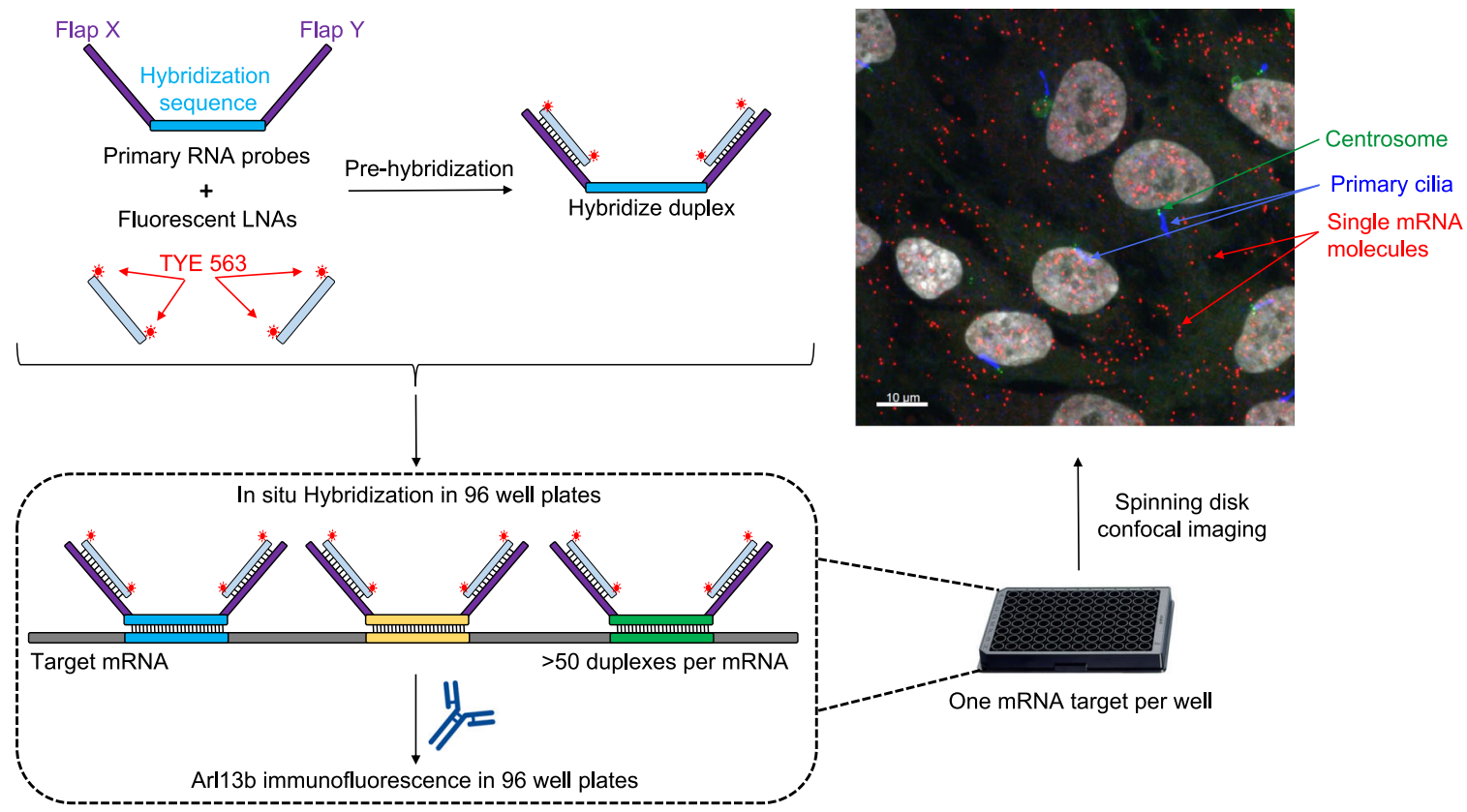

b

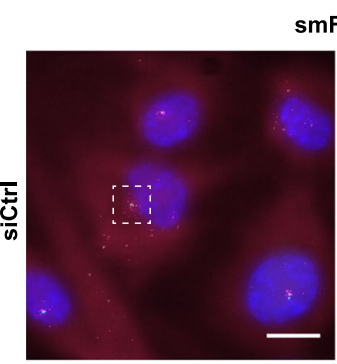

SmFISH

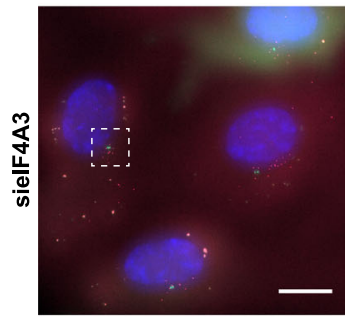

Hoechst
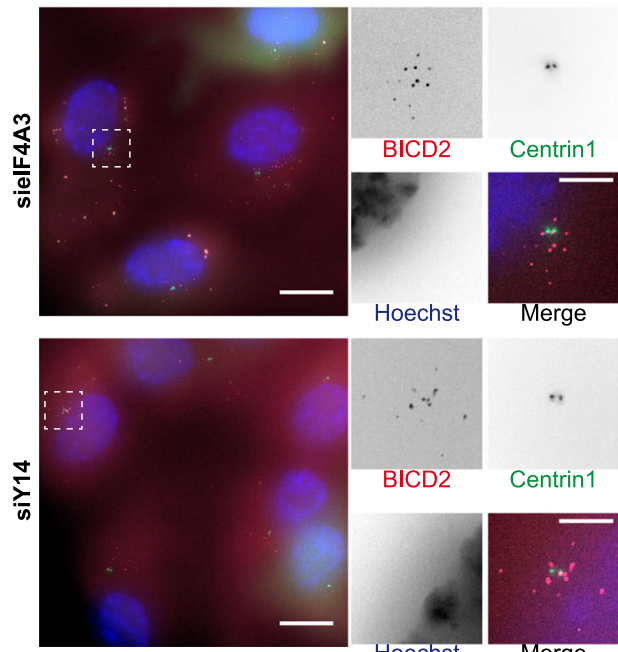

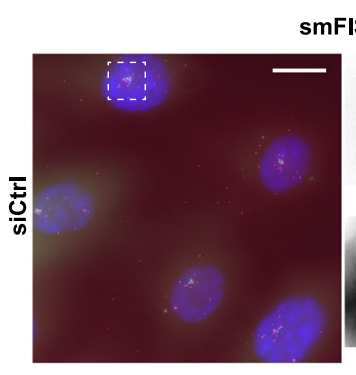

smFISH
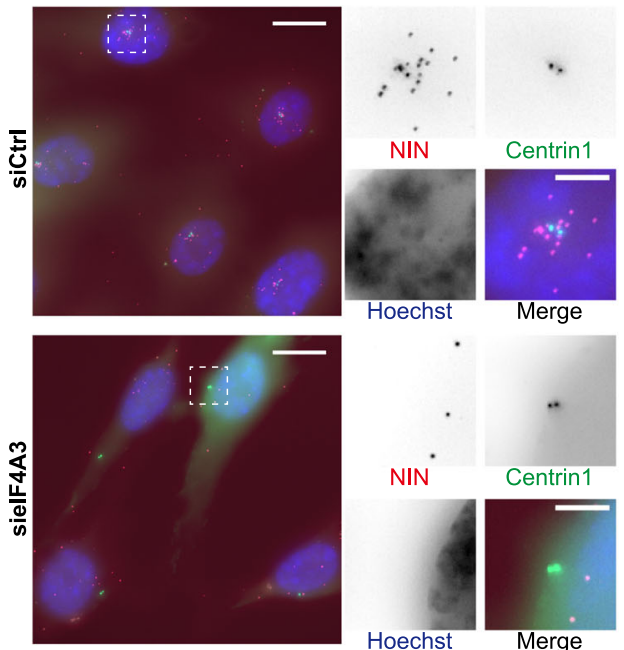

Hoechst

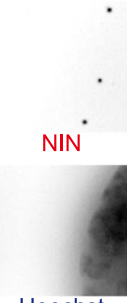

Hoechst

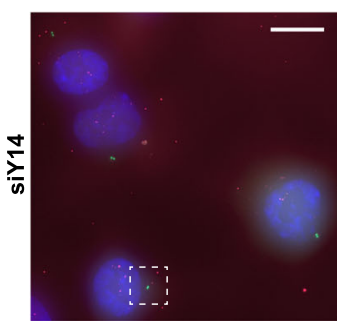

d

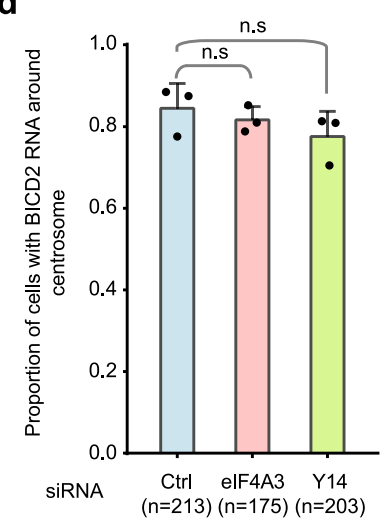

e

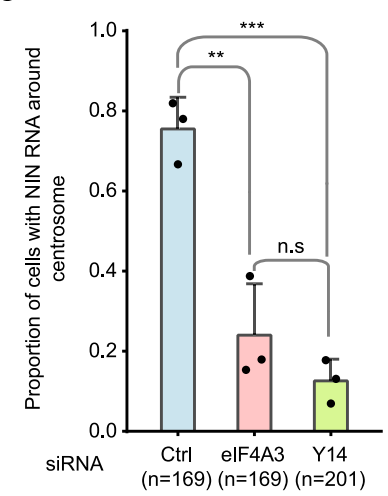

CAUUCGCUCAGCUCAUAAU-3') with Lipo2000 (Invitrogen) in Opti-MEM (Gibco). After $3 \mathrm{~h}$, cells are replaced in DMEM with FBS and processed on day 4 . If required, cells were serum-starved on day 2 .

Antibodies. Provider, clone number, catalog number, lot number, host, and dilution folds are depicted in brackets as applicable. For Western blot and RIP: $\alpha$ -

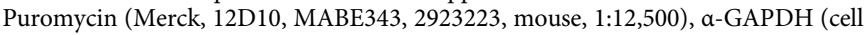
signaling, 14C10, 2118S, 14, Rabbit, 1:5000), a-eIF4A3 (affinity purified from rabbit serum $^{98}, 1: 1000$ ), $\alpha$-Y14 (affinity purified from rabbit serum ${ }^{99}, 1: 500$ ), $\alpha$-MAGOH (Santacruz, 21B12, sc-56724,C2013, mouse, 1:500), a-MLN51 (affinity purified from rabbit serum ${ }^{99}, 1: 500$ ), $\alpha$-Rab5 (affinity purified from rabbit serum ${ }^{98}$ ), $\alpha$ mouse antibody conjugated with HRP (Bethyl, A90-116P, Goat, 1:5000), and $\alpha-$ Rabbit antibody conjugated with HRP (Promega, W401B, 0000390794, Goat, 1:5000). For immunofluorescence: a-FOP (Abnova, 2B1, H00011116-M01, 112912B1, Mouse IgG2b, 1:1000), a-polyglutamylated tubulin (ADIPOGEN, GT-335, AG-20B-0020, A27791601, Mouse IgG1, 1:500), a-Y14 (Santacruz, 4C4, sc-32312, $\mathrm{H} 2416$, mouse IgG2b, 1:50), $\alpha$-eIF4A3 (affinity purified from rabbit serum ${ }^{98}$ 1:2000), $\alpha$ - $\alpha$-tubulin (Sigma, DM1A, T6199, 11M4849, mouse IgG1, 1:500), $\alpha-\beta$ tubulin (Biolegend, TU27, 903401, B203934, Mouse, 1:1000), $\alpha-\gamma$-tubulin (Sigma, GTU-88, T6557, 034M4794V, Mouse 1:500), a-EDC4 (Santacruz, SC-8418, Mouse, 1:1000), $\alpha$-DDX6 (Bethyl, BL2142, A300-461A, Rabbit, 1:1000), a-TIA-1 
Fig. 5 EJC is required for centrosomal localization of NIN mRNA. Summary of the high-throughput smiFISH pipeline (a). Top left: primary RNA probes contain a hybridization sequence that is complementary to the target mRNA flanked by two overhangs named Flap X and $\mathrm{Y}$. Each Flap was annealed to a locked nucleic acid (LNA) oligo labeled with two TYE 563 molecules in a pre-hybridization step. Bottom: Duplexes were then hybridized to the mRNA of interest followed by immunofluorescence against Arl13b to label primary cilia in 96-well plates. Plates were finally imaged with a spinning disk confocal microscope. Top right: A micrograph showing a typical field of view from the screen. Red dots correspond to a single mRNA molecule. Scale bar represents $10 \mu \mathrm{m}$. Quiescent RPE1 cells stably expressing centrin1-GFP were stained by probes against BICD2 mRNA (b) or NIN mRNA (c) after knock-down of either elF4A3 or Y14. Nuclei were stained by Hoechst. Images are resulted from maximum intensity projections of 14 z-stacks acquired at every $0.5 \mu$ m. Right panels show enlarged images of the white dashed square in the left panels. Scale bars in the left panels are $10 \mu \mathrm{m}$, and scale bars in right panels are $3 \mu \mathrm{m}$ $(\mathbf{b}, \mathbf{c})$. Proportion of cells displaying centrosomal BICD2 (d) or NIN (e) RNA pattern was depicted. Columns and bars depict mean \pm SD of three independent experiments. n.s $P>0.05,{ }^{\star \star} P \leq 0.01$, and ${ }^{\star \star \star} P \leq 0.001$, two-tailed $t$-test. The number of cells analyzed in three independent experiments is provided (d, e) Source data are provided as a Source Data file.

(Santacruz, C-20, SC-1751, Goat, 1:200), a-eIF4E (Santacruz, FL217, SC-13963, D1112, Rabbit 1:200), $\alpha$-GM130 (BD bioscience, 35GM130, BD610822, 6217559 Mouse IgG1, 1:2000), $\alpha$-pericentrin (Covance, PRB-432C, E11HF01670, Rabbit 1:500), a-PCM1 (Cell signaling, G2000, 5213S, 1, Rabbit 1:600), a-9G8 (described previously ${ }^{100}$, Rabbit 1:1000), $\alpha$-SC35 (described previously ${ }^{100}$, Mouse 1:1000), $\alpha$ NIN (Institut curie, described in previous report ${ }^{101}$, Human, 1:200), $a$-Arl13b (proteintech, 17711-1-AP, Rabbit, 1:4500), a-Goat DyLight650 (Bethyl, A50201D5, Donkey, 1:400), a-Human Alexa 488 (Invitrogen, A11013, 1173476, Goat, 1:500), a-Rabbit Alexa594 (ThermoFisher, A21207, 1107500, Donkey, 1:400), aMouse IgG1 Alexa594 (Invitrogen, A21203, 645165, Donkey, 1:500), $\alpha$-Mouse IgG2b Alexa488 (ThermoFisher, A21141, 2128994, Goat, 1:500), a-Mouse IgG1 Alexa647 (ThermoFisher, A21240, 2092265, Goat, 1:400), a-Mouse Alexa488 (Thermofischer, A-11001,1939600, Goat, 1:500), and $\alpha$-Rabbit Cy5 (Jackson ImmunoResearch, 111-176-047, 57833, Goat, 1:800).

SUnSET analysis and western blot. Cells were incubated with $4.5 \mu \mathrm{M}$ Puromycin (InvivoGen) for $15 \mathrm{~min}$ at $37^{\circ} \mathrm{C}$. After washing with PBS, cells were scraped, pelleted at $0.5 \mathrm{rcf}$ and the pellet was lysed in RIPA buffer $(20 \mathrm{mM}$ Tris-Cl pH 7.5, $150 \mathrm{mM} \mathrm{NaCl}, 1 \mathrm{mM} \mathrm{Na}{ }_{2}$ EDTA, $1 \mathrm{mM}$ EGTA, $1 \% \mathrm{NP}-40,1 \%$ sodium deoxycholate) supplemented with protease inhibitor mix (Millipore). Protein concentrations were determined by Bradford protein assay. $25 \mu \mathrm{g}$ of proteins was electrophoresed in a $12 \%$ SDS-polyacrylamide gel. Proteins were electrotransferred onto a $0.2 \mu \mathrm{m}$ nitrocelluose membrane (GE Healthcare) and blocked with $5 \%$ skim milk in $0.1 \%$ Tween 20 in tris buffer saline (T-TBS) for $30 \mathrm{~min}$ at RT. The membranes were incubated overnight at $4{ }^{\circ} \mathrm{C}$ with a-puromycin antibody in blocking solution. An a-mouse antibody conjugated with HRP was incubated for $2 \mathrm{~h}$ at RT. Puromycilated peptides were visualized by chemiluminescence with SuperSignal West Pico PLUS (Thermo Scientific). Total protein on the membrane was stained with PierceTM reversible protein stain kit (Thermo) by manufacturer's instruction. Western blot for eIF4A, Y14, and GAPDH was performed as described above with corresponding to primary and secondary antibodies.

Flow cytometry. Cells were trypsinized and resuspended with DPBS. Resuspended cells were permeabilized by incubating with extraction buffer $(0.2 \%$ Triton X-100 in PBS) for $5 \mathrm{~min}$ in ice, fixed by incubating with fixation buffer (2\% PFA in PBS) for $15 \mathrm{~min}$ in RT and stored in storage buffer (3\% FBS, $0.09 \%$ sodiumazide in DPBS). Cells were labeled by $10 \mu \mathrm{g} / \mathrm{ml}$ of HOECHST 33258 for $30 \mathrm{~min}$ in RT. After HOECHST 33258 staining, samples were analyzed on a ZE5 cell analyzer (Bio-Rad) using Everest acquisition software (Version 2.5). Data were analyzed using FlowJo software (FlowJo, LLC, version 10.6.0). Briefly, cells were selected based on their morphology (SSC versus FSC: "Cells" population) then doublets of cells were excluded based on Hoechst fluorescent parameters (Hoechst-A versus Hoechst-W: "Single cells" population). Cell cycle analysis was performed on single cell population using FlowJo module for cell cycle.

Immunofluorescence. Cells on coverslips were washed with DPBS, fixed with $4 \%$ paraformaldehyde in PBS for 10 min at RT, permeabilized with $0.1 \%$ Triton X-100 in PBS for $2 \mathrm{~min}$ at RT, blocked with $1 \%$ BSA in PBS for $30 \mathrm{~min}$ in RT, incubated for $1 \mathrm{~h}$ with primary antibodies diluted in blocking solution and nuclei were stained with HOECHST 33258, $1 \mu \mathrm{g} / \mathrm{ml}$ in blocking solution for $5 \mathrm{~min}$ at RT. Coverslips were next incubated with secondary antibodies for $1 \mathrm{~h}$ in RT and mounted with Fluoromount-G (Invitrogen) on slideglasses. For RNaseA treatment, RNase A (Sigma) was prepared by dissolving $10 \mathrm{mg} / \mathrm{ml}$ RNaseA in $10 \mathrm{mM}$ Tris-Cl $\mathrm{pH} 7.5$, $15 \mathrm{mM} \mathrm{NaCl}$. To inactivate contaminating DNases, the RNaseA solution was heated at $98^{\circ} \mathrm{C}$ for $15 \mathrm{~min}$. Coverslips were washed with PBS followed by a wash with CSK buffer (10 mM PIPES pH 7.0, $100 \mathrm{mM} \mathrm{NaCl}, 300 \mathrm{mM}$ sucrose, $3 \mathrm{mM}$ $\mathrm{MgCl}_{2}$ ) and permeabilized with $0.5 \%$ Tween- 20 for $5 \mathrm{~min}$ at RT. Coverslips were incubated with $5 \mathrm{mg} / \mathrm{ml}$ of RNaseA at $37^{\circ} \mathrm{C}$ for $15 \mathrm{~min}$ and next washed two times with PBS followed by 10 min incubation with $4 \%$ paraformaldehyde in PBS. Cells were additionally washed with PBS for three times and immunofluorescence was performed as described above.
High-throughput single molecule inexpensive fluorescent in situ hybridization with immunofluorescence (HT-smiFISH-IF) and conventional smiFISH. RPE1 cells stably expressing centrin1-GFP were seeded in 96-well glass bottom plates (SensoPlates, Greiner) and induced quiescence the next day by a $24 \mathrm{~h}$ culture in Dulbecco's modified Eagle's medium (DMEM, Gibco) with $0.25 \%$ fetal bovine serum (FBS, Sigma-Aldrich). Cells were then directly fixed for $20 \mathrm{~min}$ at RT with $4 \%$ paraformaldehyde (Electron Microscopy Sciences) diluted in PBS, and permeabilized with $70 \%$ ethanol overnight at $4{ }^{\circ} \mathrm{C}$.

To generate primary RNA probes used in the high-throughput smiFISH screen and conventional smiFISH experiments, a pool of DNA oligonucleotides (GenScript) was used. The oligonucleotide design was based on the Oligostan script $^{69}$ with each oligo having a gene-specific segment that will hybridize to the mRNA of interest, flanked by two common overhangs named Flap X and Flap Y Briefly, a first series of PCR was performed using gene-specific barcodes placed at the extremities of each oligo to amplify specific probe sets using a hot start Phusion DNA polymerase (Thermo Fisher Scientific, F549L). A second series of PCR was done to add the T7 RNA polymerase promoter using the following primers: FLAP $\mathrm{Y}$ sequence with the addition of the T7 sequence at its $5^{\prime}$ end $\left(5^{\prime}-\right.$

TAATACGACTCACTATAGGGTTACACTCGGACCTCGTCGACATGCATT$\left.3^{\prime}\right)$, and the reverse complement sequence of FLAP X (5'-

CACTGAGTCCAGCTCGAAACTTAGGAGG-3'). This PCR reaction was carried out with GoTaq G2 hot start DNA Polymerase (Promega, F549L). All PCR reactions were in 96-well plates with a Freedom EVO 200 (Tecan) robotic platform. PCR products were checked by capillary electrophoresis on a Caliper LabChip GX analyzer (PerkinElmer). The products of the second PCR were purified with a NucleoSpin 96 PCR Clean-up kit (Macherey-Nagel), lyophilized, and resuspended in DNase/RNase-free distilled water (Invitrogen). In vitro transcription was subsequently performed with T7 RNA polymerase and the obtained primary probes were analyzed by capillary electrophoresis using a Fragment Analyzer instrument (Advanced Analytical).

$50 \mathrm{ng}$ of primary probes (total amount of the pool of probes) and $25 \mathrm{ng}$ of each of the secondary probes (LNA oligonucleotides targeting FLAP X and FLAP Y labeled with TYE 563, Qiagen) were pre-hybridized in either $100 \mu$ of $1 \times$ SSC for conventional smiFISH, or in the following pre-hybridization buffer: $1 \times$ SSC, $7.5 \mathrm{M}$ urea (Sigma-Aldrich), $0.34 \mu \mathrm{g} / \mathrm{ml}$ tRNA, $10 \%$ dextran sulfate. Pre-hybridization was performed on a thermocycler with the following program: $90^{\circ} \mathrm{C}$ for $3 \mathrm{~min}, 53^{\circ} \mathrm{C}$ for $15 \mathrm{~min}$, up until probe usage. Plates with fixed cells were washed with PBS and hybridization buffer ( $1 \times$ SSC, $7.5 \mathrm{M}$ urea). For conventional smiFISH, the prehybridized mixture was diluted in the same pre-hybridization buffer as above. Hybridization was then carried out overnight at $48^{\circ} \mathrm{C}$. The next day, plates were washed eight (screen) or three (conventional smiFISH) times for 20 min each in $1 \times$ SSC $7.5 \mathrm{M}$ urea at $48^{\circ} \mathrm{C}$, followed by three PBS rinses. The samples that were not processed into immunofluorescence were directly mounted on slide glass with Vectashield mounting medium with DAPI (Vector laboratories).

For post HT-smiFISH immunofluorescence, cells were permeabilized with $0.1 \%$ Triton-X100 in PBS for $10 \mathrm{~min}$ at room temperature and washed twice with PBS. For cilia labeling, plates were incubated overnight at $4^{\circ} \mathrm{C}$ with an anti-Arll3b antibody diluted in $0.1 \%$ Triton X-100 PBS. The next day, plates were washed three times with PBS, and incubated with a Cy5-labeled goat anti-rabbit secondary antibody in $0.1 \%$ Triton X-100 PBS. After $2 \mathrm{~h}$ of incubation at room temperature, plates were washed three times with PBS. To label DNA, cells were then stained with $1 \mu \mathrm{g} / \mathrm{ml}$ DAPI diluted in PBS, and finally mounted in 90\% glycerol (VWR), $1 \mathrm{mg} / \mathrm{ml}$ p-phenylenediamine (Sigma-Aldrich), PBS pH 8.

Sequences of smFISH probes in high-throughput smFISH screen are provided in Supplementary Data 2.

RIP analysis and RT-qPCR. Antibody conjugated beads were prepared by incubating $20 \mu \mathrm{l}$ of protein A Dynabeads (novex) with $20 \mu \mathrm{g}$ of corresponding antibodies for $30 \mathrm{~min}$ in RT. After washing two times with PXL buffer (1× PBS, $0.1 \%$ SDS, $0.5 \%$ NP40, $0.5 \%$ sodiumdeoxycholate), beads were blocked by incubation in PXL buffer supplemented with $100 \mu \mathrm{g} / \mathrm{ml}$ of BSA (Sigma) followed by two times of wash with PXL buffer. Quiescent RPE1 cells were lysed in PXL buffer supplemented with protease inhibitor cocktail set III (Merck), RNase free RQ1 DNase (Promega), and 
a

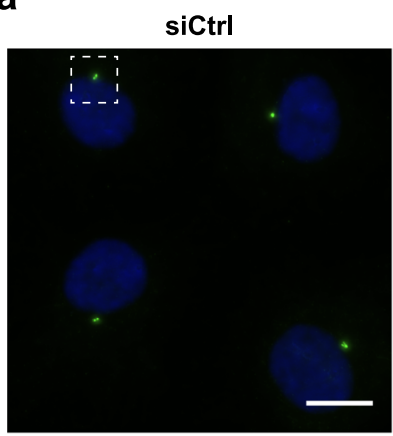

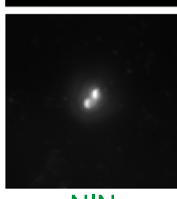

NIN

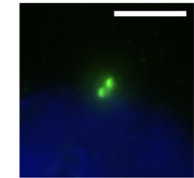

Merge
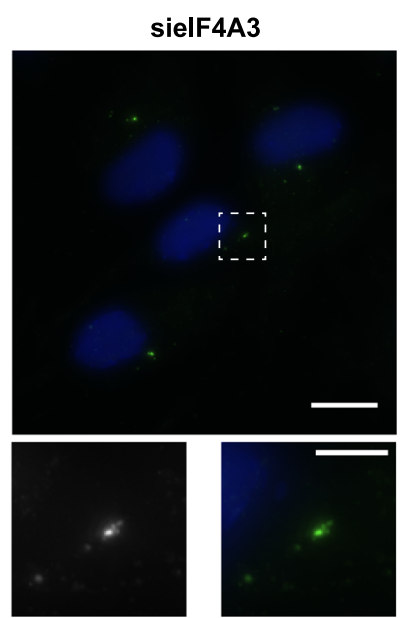

NIN

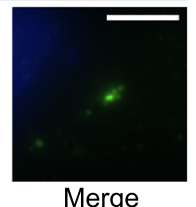

Merge

C

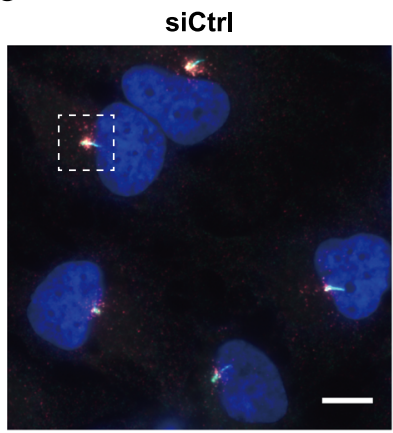

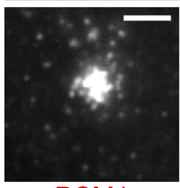

PCM1

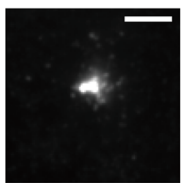

FOP

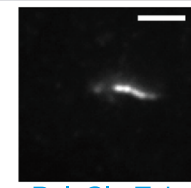

PolyGlu-Tub

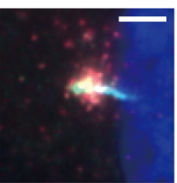

Merge

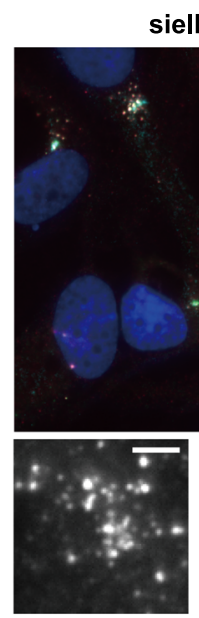

PCM1

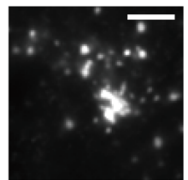

FOP
sielF4A3

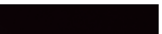

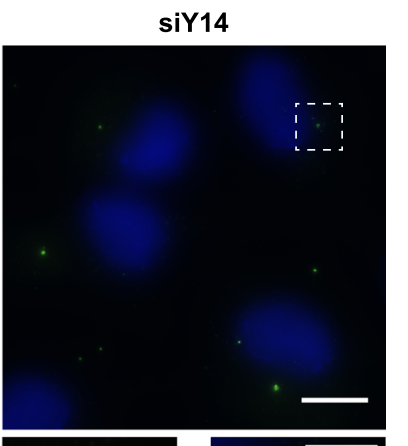

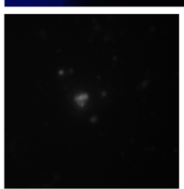

NIN

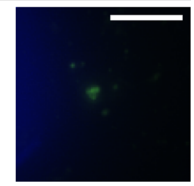

Merge b

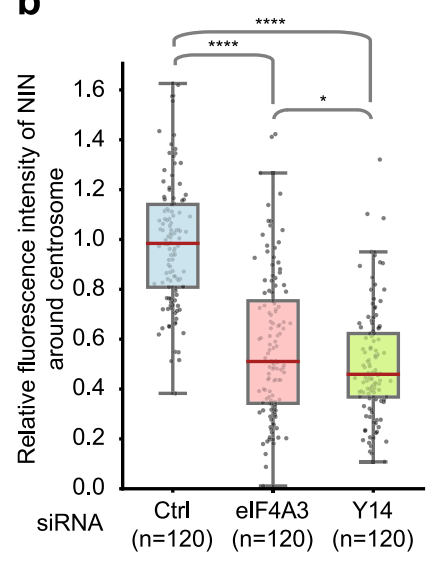

d

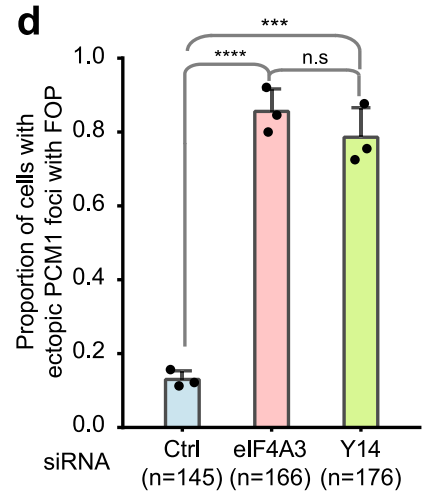

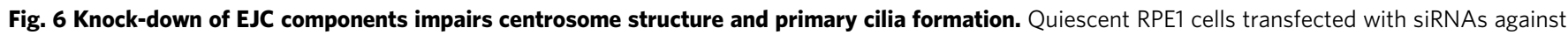
elF4A3 or Y14 were stained for NIN (a) or PCM1 (centriolar satellite protein), FOP, and poly-glutamylated tubulin (PolyGlu-Tub; c). Nuclei were stained by Hoechst. Lower panels are enlarged images marked by white dashed square in the upper panels. Scale bars in the upper and lower panels are 10 and $3 \mu$, respectively $(\mathbf{a}, \mathbf{c})$. Images were processed by maximum intensity projections of 15 z-stacks acquired at every $0.5 \mu \mathrm{m}$ (a). Quantification of fluorescence intensities of NIN were performed as described in the legend of Fig. 1. Boxes represent values between the 25th lower and 75th higher percentile, and the red lines mark the median. Whiskers above and below correspond to 0.35th lower and 99.65th higher percentile, respectively. The average fluorescence intensity of NIN in Ctrl siRNA treated cells is set to 1.0 (b). Proportion of cells with ectopic centriolar satellite with FOP upon the siRNA treatments indicated (d). Proportion of ciliated cells upon the indicated siRNA treatments (e). Columns and bars depict mean \pm SD of three independent experiments (d, e). n.s. $P>0.05,{ }^{\star} P \leq 0.05,{ }^{\star \star} P \leq 0.01,{ }^{\star \star \star} P \leq 0.001$, and ${ }^{\star \star \star \star} P \leq 0.0001$, two-tailed Mann-Whitney test (b) and two-tailed $t$-test (d, e). The number of cells analyzed in three independent experiments is provided (b, d, e). Source data are provided as a Source Data file.

recombinant ribonuclease inhibitor (Promega) followed by $10 \mathrm{~min}$ incubation in ice. Lysates were centrifuged at $16,000 \mathrm{rcf}$ in $4{ }^{\circ} \mathrm{C}$ for $10 \mathrm{~min}$. $10 \%$ of supernatant was kept for quantification of input RNAs, and $90 \%$ of lysates were incubated with antibody-conjugated protein A Dynabeads for overnight in $4{ }^{\circ} \mathrm{C}$. Beads were washed three times with IP500 buffer $(10 \mathrm{mM}$ Tris-Cl pH 7.5, $500 \mathrm{mM} \mathrm{NaCl}, 2.5 \mathrm{mM}$ $\left.\mathrm{MgCl}_{2}, 1 \% \mathrm{NP} 40\right)$ followed by two times of wash with IP150 buffer (10 mM Tris-Cl $\left.\mathrm{pH} 7.5,150 \mathrm{mM} \mathrm{NaCl}, 2.5 \mathrm{mM} \mathrm{MgCl}_{2}, 1 \% \mathrm{NP} 40\right)$. Input and beads supplemented with up to $100 \mu \mathrm{l}$ of IP 150 buffer were vortexed after adding $100 \mu \mathrm{l}$ phenol chloroform $\mathrm{pH} 4.5$. After $5 \mathrm{~min}$ of centrifugation at $16,000 \mathrm{rcf}$ in $4{ }^{\circ} \mathrm{C}$, aqueous phase is proceeded to ethanol precipitation. Pellets were dissolved in pre-heated $\left(55^{\circ} \mathrm{C}\right)$ water followed by $30 \mathrm{~min}$ of RQ1 DNase treatment in $37^{\circ} \mathrm{C}$. Phenol extraction and ethanol precipitation performed again as described above. For general RT-qPCR, RNA was purified by Monarch Total RNA Miniprep kit (New england BioLabs). Reverse transcription was performed with superscript IV (Invitrogen) according to manufacturer's instruction. For BICD2 and NIN mRNAs in RIP analysis, gene-specific primers were used for reverse transcription. Intronless gene for RIP analysis and RNAs for general RT-qPCR are reverse transcribed with Oligo dT (Thermo Scientific) and random hexamer (Thermo Scientific), respectively. qPCR was performed by using PowerUP SYBR Green Master Mix (appliedbiosystems) on CFX384 Real time system (Bio-Rad). \% input was calculated 


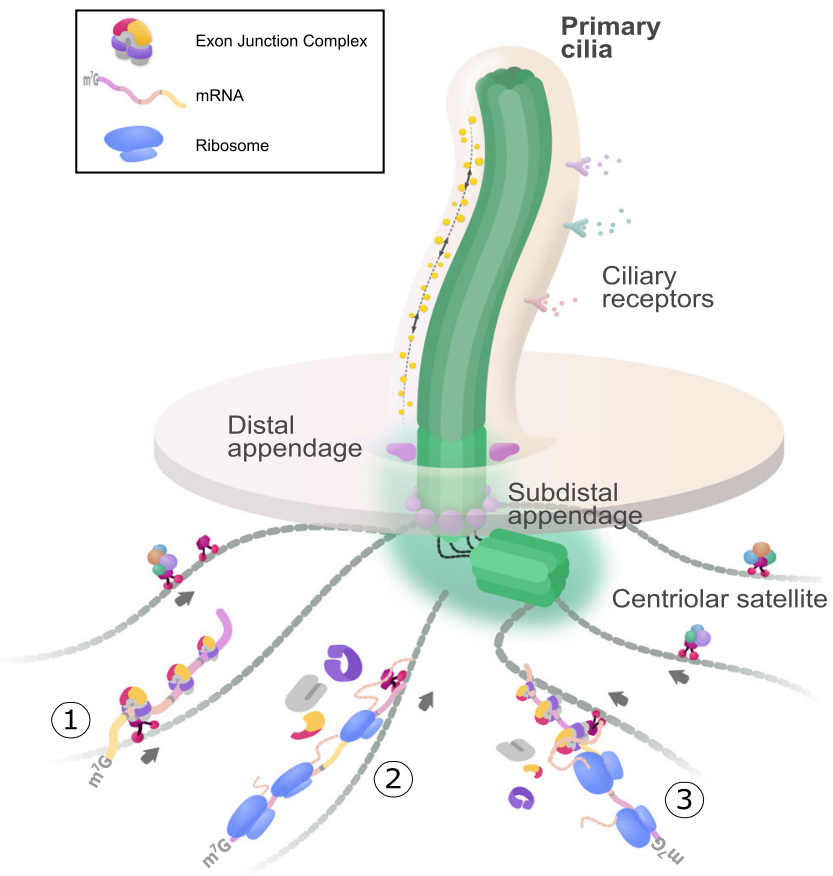

Fig. 7 Multiple pathways for RNA localization toward centrosome. During quiescence, mother centriole tightly attaches to plasma membrane by distal appendages to nucleate the formation of primary cilia. To relay the signals from environment, several receptors are embedded at ciliary membrane. Centriolar satellites deliver components of centrosome and primary cilia. Centrosomal transcripts could be localized by (1) EJC and/or other RNPsmediated pathway, (2) polysome-dependent pathway, and (3) polysomedependent and EJC-dependent pathway.

based on threshhold cycle (Cq) of input and RIP RNAs, and relative RNA level in total lysates were normalized by housekeeping gene GAPDH. Primers for reverse transcription and qPCR are described in Supplementary Table S1.

Fluorescence microscopy and image analysis. Images were acquired with an epifluorescence microscope (Nikon ECLIPSE Ti) equipped with a plan APO VC $\times 60$ objective (NA 1.4, Nikon), CCD camera (ORCA Flash 4.0, Hamamatsu), and operated by Micro-Manager (MM studio, version 1.4.22). Maximum intensity projection of $\mathrm{z}$-stacks was processed by Fiji. HT-smiFISH-IF were imaged on an Opera Phenix High-Content Screening System (PerkinElmer), with a $\times 63$ waterimmersion objective (NA 1.15).

Centrosomes are detected by poly-glutamylated tubulin and/or FOP staining. Cilia are detected poly-glutamylated tubulin staining. In multiciliated ependymal cell, centrioles were chosen at random in multiciliated ependymal cell. P-bodies are identified by both of DDX6 and EDC4. The number of P-body per cell was determined in images that are processed by maximum intensity projection from 6 $z$-stacks acquired at every $1 \mu \mathrm{m}$. Proportion of ciliated cells and fraction of cells with detectable eIF4A3 and Y14 are determined by vision-based manual annotation from acquired images and calculated. Fluorescence intensities were measured in $2 \mu \mathrm{m}$ diameter circles around centrosomes (RPE1, or mouse NSC) or base of cilia (ependymal cells) were determined by Image J. Centrosomes and base of cilia that do not overlap the nucleus were selected to exclude nuclear background interference. The background was defined as the lowest pixel intensity in the circle and subtracted from the average fluorescence intensity. Pearson's correlation coefficient for colocalization was determined in the selected area by Coloc2 plugin in Image (National Institute of Health, Bethesda, version 1.52d). Box plots and bar graphs are made by using matplotlib and GraphPad Prism 7, respectively.

Reporting summary. Further information on research design is available in the Nature Research Reporting Summary linked to this article.

\section{Data availability}

The data provided in this study are available from the corresponding author upon reasonable request. Requests for unique biological materials such as antibodies or cell lines should be directed to the corresponding author. Sequence information of mRNAs used in high throughput smFISH screen is provided in Supplementary Data 1 as ensemble access codes (https://www.ensembl.org/). Source data are provided with this paper.

Received: 25 April 2020; Accepted: 14 January 2021; Published online: 01 March 2021

\section{References}

1. Singh, G., Pratt, G., Yeo, G. W. \& Moore, M. J. The clothes make the mRNA: past and present trends in mRNP fashion. Annu. Rev. Biochem. 84, 325-354 (2015).

2. Kwon, S. C. et al. The RNA-binding protein repertoire of embryonic stem cells. Nat. Struct. Mol. Biol. 20, 1122-1130 (2013).

3. Castello, A. et al. Insights into RNA biology from an atlas of mammalian mRNA-binding proteins. Cell 149, 1393-1406 (2012).

4. Baltz, A. G. et al. The mRNA-bound proteome and its global occupancy profile on protein-coding transcripts. Mol. Cell 46, 674-690 (2012).

5. Gehring, N. H., Wahle, E. \& Fischer, U. Deciphering the mRNP code: RNAbound determinants of post-transcriptional gene regulation. Trends Biochem. Sci. 42, 369-382 (2017).

6. Hentze, M. W., Castello, A., Schwarzl, T. \& Preiss, T. A brave new world of RNA-binding proteins. Nat. Rev. Mol. Cell Biol. 19, 327-341 (2018).

7. Adivarahan, S. et al. Spatial organization of single mRNPs at different stages of the gene expression pathway. Mol. Cell 72, 727-738 (2018). e725.

8. Metkar, M. et al. Higher-order organization principles of pre-translational mRNPs. Mol. Cell 72, 715-726 (2018). e713.

9. Sauliere, J. et al. CLIP-seq of eIF4AIII reveals transcriptome-wide mapping of the human exon junction complex. Nat. Struct. Mol. Biol. 19, 1124-1131 (2012).

10. Singh, G. et al. The cellular EJC interactome reveals higher-order mRNP structure and an EJC-SR protein nexus. Cell 151, 750-764 (2012).

11. Tange, T. O., Shibuya, T., Jurica, M. S. \& Moore, M. J. Biochemical analysis of the EJC reveals two new factors and a stable tetrameric protein core. RNA 11, 1869-1883 (2005).

12. Ballut, L. et al. The exon junction core complex is locked onto RNA by inhibition of eIF4AIII ATPase activity. Nat. Struct. Mol. Biol. 12, 861-869 (2005).

13. Andersen, C. B. et al. Structure of the exon junction core complex with a trapped DEAD-box ATPase bound to RNA. Science 313, 1968-1972 (2006).

14. Bono, F., Ebert, J., Lorentzen, E. \& Conti, E. The crystal structure of the exon junction complex reveals how it maintains a stable grip on mRNA. Cell 126, 713-725 (2006).

15. Le Hir, H., Sauliere, J. \& Wang, Z. The exon junction complex as a node of post-transcriptional networks. Nat. Rev. Mol. Cell Biol. 17, 41-54 (2016).

16. Gehring, N. H., Lamprinaki, S., Kulozik, A. E. \& Hentze, M. W. Disassembly of exon junction complexes by PYM. Cell 137, 536-548 (2009).

17. Boehm, V. \& Gehring, N. H. Exon junction complexes: supervising the gene expression assembly line. Trends Genet. 32, 724-735 (2016).

18. Nott, A., Le Hir, H. \& Moore, M. J. Splicing enhances translation in mammalian cells: an additional function of the exon junction complex. Genes Dev. 18, 210-222 (2004).

19. Chazal, P. E. et al. EJC core component MLN51 interacts with eIF3 and activates translation. Proc. Natl Acad. Sci. USA 110, 5903-5908 (2013).

20. Kurosaki, T., Popp, M. W. \& Maquat, L. E. Quality and quantity control of gene expression by nonsense-mediated mRNA decay. Nat. Rev. Mol. Cell Biol. 20, 406-420 (2019).

21. Wang, T. et al. Identification and characterization of essential genes in the human genome. Science 350, 1096-1101 (2015).

22. Micklem, D. R. et al. The mago nashi gene is required for the polarisation of the oocyte and the formation of perpendicular axes in Drosophila. Curr. Biol. 7, 468-478 (1997)

23. Newmark, P. A., Mohr, S. E., Gong, L. \& Boswell, R. E. mago nashi mediates the posterior follicle cell-to-oocyte signal to organize axis formation in Drosophila. Development 124, 3197-3207 (1997).

24. Kawano, T., Kataoka, N., Dreyfuss, G. \& Sakamoto, H. Ce-Y14 and MAG-1, components of the exon-exon junction complex, are required for embryogenesis and germline sexual switching in Caenorhabditis elegans. Mech. Dev. 121, 27-35 (2004)

25. van der Weele, C. M., Tsai, C. W. \& Wolniak, S. M. Mago nashi is essential for spermatogenesis in Marsilea. Mol. Biol. Cell 18, 3711-3722 (2007).

26. Wittkopp, N. et al. Nonsense-mediated mRNA decay effectors are essential for zebrafish embryonic development and survival. Mol. Cell. Biol. 29, 3517-3528 (2009).

27. Haremaki, T., Sridharan, J., Dvora, S. \& Weinstein, D. C. Regulation of vertebrate embryogenesis by the exon junction complex core component Eif4a3. Dev. Dyn. 239, 1977-1987 (2010). 
28. Pascuan, C., Frare, R., Alleva, K., Ayub, N. D. \& Soto, G. mRNA biogenesisrelated helicase eIF4AIII from Arabidopsis thaliana is an important factor for abiotic stress adaptation. Plant Cell Rep. 35, 1205-1208 (2016).

29. McMahon, J. J., Miller, E. E. \& Silver, D. L. The exon junction complex in neural development and neurodevelopmental disease. Int. J. Dev. Neurosci. 55, 117-123 (2016).

30. Albers, C. A. et al. Compound inheritance of a low-frequency regulatory SNP and a rare null mutation in exon-junction complex subunit RBM8A causes TAR syndrome. Nat. Genet. 44, S431-S432 (2012). 435-439.

31. Favaro, F. P. et al. A noncoding expansion in EIF4A3 causes Richieri-CostaPereira syndrome, a craniofacial disorder associated with limb defects. Am. J. Hum. Genet. 94, 120-128 (2014).

32. Nguyen, L. S. et al. Contribution of copy number variants involving nonsensemediated mRNA decay pathway genes to neuro-developmental disorders. Hum. Mol. Genet. 22, 1816-1825 (2013).

33. Silver, D. L. et al. The exon junction complex component Magoh controls brain size by regulating neural stem cell division. Nat. Neurosci. 13, 551-558 (2010).

34. McMahon, J. J., Shi, L. \& Silver, D. L. Generation of a Magoh conditional allele in mice. Genesis 52, 752-758 (2014).

35. Pilaz, L. J. et al. Prolonged mitosis of neural progenitors alters cell fate in the developing brain. Neuron 89, 83-99 (2016).

36. Mao, H. et al. Rbm8a haploinsufficiency disrupts embryonic cortical development resulting in microcephaly. J. Neurosci. 35, 7003-7018 (2015).

37. Mao, H., McMahon, J. J., Tsai, Y. H., Wang, Z. \& Silver, D. L. Haploinsufficiency for core exon junction complex components disrupts embryonic neurogenesis and causes p53-mediated microcephaly. PLoS Genet. 12, e1006282 (2016).

38. Mao, H., Brown, H. E. \& Silver, D. L. Mouse models of Casc3 reveal developmental functions distinct from other components of the exon junction complex. RNA 23, 23-31 (2017)

39. Jolly, L. A., Homan, C. C., Jacob, R., Barry, S. \& Gecz, J. The UPF3B gene, implicated in intellectual disability, autism, ADHD and childhood onset schizophrenia regulates neural progenitor cell behaviour and neuronal outgrowth. Hum. Mol. Genet. 22, 4673-4687 (2013).

40. Alrahbeni, T. et al. Full UPF3B function is critical for neuronal differentiation of neural stem cells. Mol. Brain 8, 33 (2015).

41. Lou, C. H. et al. Posttranscriptional control of the stem cell and neurogenic programs by the nonsense-mediated RNA decay pathway. Cell Rep. 6, 748-764 (2014).

42. Conduit, P. T., Wainman, A. \& Raff, J. W. Centrosome function and assembly in animal cells. Nat. Rev. Mol. Cell Biol. 16, 611-624 (2015).

43. Delgehyr, N. et al. Ependymal cell differentiation, from monociliated to multiciliated cells. Methods Cell Biol. 127, 19-35 (2015).

44. Spassky, N. et al. Adult ependymal cells are postmitotic and are derived from radial glial cells during embryogenesis. J. Neurosci. 25, 10-18 (2005).

45. Spassky, N. \& Meunier, A. The development and functions of multiciliated epithelia. Nat. Rev. Mol. Cell Biol. 18, 423-436 (2017).

46. Acquaviva, C. et al. The centrosomal FOP protein is required for cell cycle progression and survival. Cell Cycle 8, 1217-1227 (2009).

47. Stearns, T. \& Winey, M. The cell center at 100. Cell 91, 303-309 (1997).

48. Bobinnec, Y. et al. Centriole disassembly in vivo and its effect on centrosome structure and function in vertebrate cells. J. Cell Biol. 143, 1575-1589 (1998).

49. Palacios, I. M., Gatfield, D., St Johnston, D. \& Izaurralde, E. An eIF4AIII containing complex required for mRNA localization and nonsense-mediated mRNA decay. Nature 427, 753-757 (2004).

50. Kataoka, N. et al. Pre-mRNA splicing imprints mRNA in the nucleus with a novel RNA-binding protein that persists in the cytoplasm. Mol. Cell 6, 673-682 (2000).

51. Daguenet, E. et al. Perispeckles are major assembly sites for the exon junction core complex. Mol. Biol. Cell 23, 1765-1782 (2012).

52. Molla-Herman, A. et al. The ciliary pocket: an endocytic membrane domain at the base of primary and motile cilia. J. Cell Sci. 123, 1785-1795 (2010).

53. Ghossoub, R. et al. Septins 2, 7 and 9 and MAP4 colocalize along the axoneme in the primary cilium and control ciliary length. J. Cell Sci. 126, 2583-2594 (2013).

54. Fribourg, S., Gatfield, D., Izaurralde, E. \& Conti, E. A novel mode of RBDprotein recognition in the Y14-Mago complex. Nat. Struct. Biol. 10, 433-439 (2003).

55. Gong, P., Zhao, M. \& He, C. Slow co-evolution of the MAGO and Y14 protein families is required for the maintenance of their obligate heterodimerization mode. PLoS ONE 9, e84842 (2014).

56. Degot, S. et al. Association of the breast cancer protein MLN51 with the exon junction complex via its speckle localizer and RNA binding module. J. Biol. Chem. 279, 33702-33715 (2004).

57. Spalluto, C., Wilson, D. I. \& Hearn, T. Evidence for reciliation of RPE1 cells in late G1 phase, and ciliary localisation of cyclin B1. FEBS Open Bio 3, 334-340 (2013).
58. Eisinger-Mathason, T. S. et al. Codependent functions of RSK2 and the apoptosis-promoting factor TIA-1 in stress granule assembly and cell survival. Mol. Cell 31, 722-736 (2008).

59. Dowling, R. J. et al. mTORC1-mediated cell proliferation, but not cell growth, controlled by the 4E-BPs. Science 328, 1172-1176 (2010).

60. Vander Haar, E., Lee, S. I., Bandhakavi, S., Griffin, T. J. \& Kim, D. H. Insulin signalling to mTOR mediated by the Akt/PKB substrate PRAS40. Nat. Cell Biol. 9, 316-323 (2007).

61. Bilanges, B. et al. Tuberous sclerosis complex proteins 1 and 2 control serumdependent translation in a TOP-dependent and -independent manner. Mol. Cell. Biol. 27, 5746-5764 (2007).

62. Schmidt, E. K., Clavarino, G., Ceppi, M. \& Pierre, P. SUnSET, a nonradioactive method to monitor protein synthesis. Nat. Methods 6, 275-277 (2009).

63. Standart, N. \& Weil, D. P-Bodies: cytosolic droplets for coordinated mRNA storage. Trends Genet. 34, 612-626 (2018).

64. Martin, K. C. \& Ephrussi, A. mRNA localization: gene expression in the spatial dimension. Cell 136, 719-730 (2009).

65. Chin, A. \& Lecuyer, E. RNA localization: making its way to the center stage. Biochim. Biophys. Acta Gen. Subj. 1861, 2956-2970 (2017).

66. Ryder, P. V. \& Lerit, D. A. RNA localization regulates diverse and dynamic cellular processes. Traffic 19, 496-502 (2018).

67. Panic, M., Hata, S., Neuner, A. \& Schiebel, E. The centrosomal linker and microtubules provide dual levels of spatial coordination of centrosomes. PLoS Genet. 11, e1005243 (2015)

68. Safieddine, A. et al. A choreography of centrosomal mRNAs reveals a conserved localization mechanism involving active polysome transport. Nat Commun. https://doi.org/10.1038/s41467-021-21585-7 (2021).

69. Tsanov, N. et al. smiFISH and FISH-quant-a flexible single RNA detection approach with super-resolution capability. Nucleic Acids Res. 44, e165 (2016).

70. Hoffmeister, H. et al. CHD3 and CHD4 form distinct NuRD complexes with different yet overlapping functionality. Nucleic Acids Res. 45, 10534-10554 (2017).

71. Bertran, M. T. et al. Nek9 is a Plk1-activated kinase that controls early centrosome separation through Nek6/7 and Eg5. EMBO J. 30, 2634-2647 (2011).

72. Olenick, M. A. \& Holzbaur, E. L. F. Dynein activators and adaptors at a glance. J. Cell Sci. 132 https://doi.org/10.1242/jcs.227132 (2019).

73. Delgehyr, N., Sillibourne, J. \& Bornens, M. Microtubule nucleation and anchoring at the centrosome are independent processes linked by ninein function. J. Cell Sci. 118, 1565-1575 (2005)

74. Mazo, G., Soplop, N., Wang, W. J., Uryu, K. \& Tsou, M. F. Spatial control of primary ciliogenesis by subdistal appendages alters sensation-associated properties of cilia. Dev. Cell 39, 424-437 (2016).

75. Sepulveda, G. et al. Co-translational protein targeting facilitates centrosomal recruitment of PCNT during centrosome maturation in vertebrates. Elife 7 https://doi.org/10.7554/eLife.34959 (2018).

76. Chouaib, R. et al. A Dual Protein-mRNA localization screen reveals compartmentalized translation and widespread co-translational RNA targeting. Dev. Cell https://doi.org/10.1016/j.devcel.2020.07.010 (2020).

77. Mabin, J. W. et al. The exon junction complex undergoes a compositional switch that alters mRNP structure and nonsense-mediated mRNA decay activity. Cell Rep. 25, 2431-2446 (2018). e2437.

78. Dammermann, A. \& Merdes, A. Assembly of centrosomal proteins and microtubule organization depends on PCM-1. J. Cell Biol. 159, 255-266 (2002).

79. Hartman, H., Puma, J. P. \& Gruney, T. Jr. Evidence for the association of RNA with the ciliary basal bodies of Tetrahymena. J. Cell Sci. 16, 241-259 (1974).

80. Dippell, R. V. Effects of nuclease and protease digestion on the ultrastructure of Paramecium basal bodies. J. Cell Biol. 69, 622-637 (1976).

81. Lambert, J. D. \& Nagy, L. M. Asymmetric inheritance of centrosomally localized mRNAs during embryonic cleavages. Nature 420, 682-686 (2002).

82. Groisman, I. et al. CPEB, maskin, and cyclin B1 mRNA at the mitotic apparatus: implications for local translational control of cell division. Cell 103, 435-447 (2000).

83. Buxbaum, A. R., Haimovich, G. \& Singer, R. H. In the right place at the right time: visualizing and understanding mRNA localization. Nat. Rev. Mol. Cell Biol. 16, 95-109 (2015)

84. Pichon, X. et al. Visualization of single endogenous polysomes reveals the dynamics of translation in live human cells. J. Cell Biol. 214, 769-781 (2016)

85. Wu, B., Eliscovich, C., Yoon, Y. J. \& Singer, R. H. Translation dynamics of single mRNAs in live cells and neurons. Science 352, 1430-1435 (2016).

86. Cui, X. A. \& Palazzo, A. F. Localization of mRNAs to the endoplasmic reticulum. Wiley Interdiscip. Rev. RNA 5, 481-492 (2014).

87. Redwine, W. B. et al. The human cytoplasmic dynein interactome reveals novel activators of motility. Elife 6 https://doi.org/10.7554/eLife.28257 (2017). 
88. Ghosh, S., Marchand, V., Gaspar, I. \& Ephrussi, A. Control of RNP motility and localization by a splicing-dependent structure in oskar mRNA. Nat. Struct. Mol. Biol. 19, 441-449 (2012).

89. Kugler, J. M. \& Lasko, P. Localization, anchoring and translational control of oskar, gurken, bicoid and nanos mRNA during Drosophila oogenesis. Fly 3, 15-28 (2009).

90. Breslow, D. K. \& Holland, A. J. Mechanism and regulation of centriole and cilium biogenesis. Annu. Rev. Biochem. 88, 691-724 (2019).

91. Malicki, J. J. \& Johnson, C. A. The cilium: cellular antenna and central processing unit. Trends Cell Biol. 27, 126-140 (2017).

92. Hori, A. \& Toda, T. Regulation of centriolar satellite integrity and its physiology. Cell. Mol. Life Sci. 74, 213-229 (2017).

93. Odabasi, E., Gul, S., Kavakli, I. H. \& Firat-Karalar, E. N. Centriolar satellites are required for efficient ciliogenesis and ciliary content regulation. $E M B O$ Rep. 20 https://doi.org/10.15252/embr.201947723 (2019).

94. Saade, M., Blanco-Ameijeiras, J., Gonzalez-Gobartt, E. \& Marti, E. A centrosomal view of CNS growth. Development 145 https://doi.org/10.1242/ dev.170613 (2018).

95. Jayaraman, D., Bae, B. I. \& Walsh, C. A. The genetics of primary microcephaly. Annu. Rev. Genom. Hum. Genet. 19, 177-200 (2018).

96. Wang, X. et al. Asymmetric centrosome inheritance maintains neural progenitors in the neocortex. Nature 461, 947-955 (2009).

97. Jeong, A. L. et al. Oncoprotein CIP2A promotes the disassembly of primary cilia and inhibits glycolytic metabolism. EMBO Rep. 19, https://doi.org/ 10.15252/embr.201745144 (2018).

98. Barbosa, I. et al. Human CWC22 escorts the helicase eIF4AIII to spliceosomes and promotes exon junction complex assembly. Nat. Struct. Mol. Biol. 19, 983-990 (2012).

99. Wang, Z., Murigneux, V. \& Le Hir, H. Transcriptome-wide modulation of splicing by the exon junction complex. Genome Biol. 15, 551 (2014).

100. Cavaloc, Y., Bourgeois, C. F., Kister, L. \& Stevenin, J. The splicing factors 9G8 and SRp20 transactivate splicing through different and specific enhancers. RNA 5, 468-483 (1999).

101. Bouckson-Castaing, V. et al. Molecular characterisation of ninein, a new coiled-coil protein of the centrosome. J. Cell Sci. 109, 179-190 (1996).

\section{Acknowledgements}

We thank Alice Lebreton and Dominique Weil for antibodies and Alexandre Benmerah for RPE1 cells. We thank Olivier Bensaude for critical readings of the manuscript and scientific discussions, and Nathalie Delgehyr for scientific inputs. This study was supported by the Agence Nationale de la Recherche (ANR), with the grants differEnJCe (ANR-13-BSV8-0023), spEJCificity (ANR-17-CE12-0021) to H.L.H., Hi-FISH (ANR-14CE10-30) to E.B., by Fondation pour la Recherche Médicale (FRM; grant bioinformatics) to E.B., by Ligue Nationale Contre le Cancer (LNCC) to E.B. (equipe labélisée), by the European Commission (Marie Curie ITN RNPnet) to H.L.H., the European Research Council (ERC Consolidator grant 647466) to N.S., by the program «Investissements d'Avenir» launched by the French Government and implemented by ANR (ANR-1-0LABX-54 MEMOLIFE and ANR-10-IDEX-0001-02 PSL* Research University) to H.L.H. and N.S., and by continuous financial support from the Centre National de Recherche Scientifique, the Ecole Normale Supérieure and the Institut National de la Santé et de la Recherche Médicale, France.

\section{Author contributions}

H.L.H., N.S., and O.S.K. conceived the project. R.M. and M.F. prepared primary cell cultures of mNSC and their differentiation into ependymal cells. O.S.K., R.M., and M.F. performed immunofluorescence microscopy on mNSC. O.S.K. conducted immunofluorescence microscopy on RPE1 cells. I.B. and O.S.K. performed Western blotting and Q.A. performed RIP. A.S. and E.C. performed high-throughput smFISH screen. O.S.K. performed RT-qPCR, microtubule regrowth assays, SUnSET analyses, flow cytometry experiments, smFISH experiments, all RPE1 treatments, and image analyses including quantifications. E.B., H.L.H., N.S., O.S.K., and R.M. analyzed the data. O.S.K. prepared the Figures. O.S.K. and H.L.H. wrote the manuscript that was reviewed and edited by E.B. and N.S.

\section{Competing interests}

The authors declare no competing interests.

\section{Additional information}

Supplementary information The online version contains supplementary material available at https://doi.org/10.1038/s41467-021-21590-w.

Correspondence and requests for materials should be addressed to H.L.H.

Peer review information Nature Communications thanks Louis-Jan Pilaz and other, anonymous, reviewers for their contributions to the peer review of this work.

Reprints and permission information is available at http://www.nature.com/reprints

Publisher's note Springer Nature remains neutral with regard to jurisdictional claims in published maps and institutional affiliations.

Open Access This article is licensed under a Creative Commons Attribution 4.0 International License, which permits use, sharing, adaptation, distribution and reproduction in any medium or format, as long as you give appropriate credit to the original author(s) and the source, provide a link to the Creative Commons license, and indicate if changes were made. The images or other third party material in this article are included in the article's Creative Commons license, unless indicated otherwise in a credit line to the material. If material is not included in the article's Creative Commons license and your intended use is not permitted by statutory regulation or exceeds the permitted use, you will need to obtain permission directly from the copyright holder. To view a copy of this license, visit http://creativecommons.org/ licenses/by/4.0/.

(C) The Author(s) 2021 